\title{
Automatic Red-Channel Underwater Image Restoration
}

\author{
Adrian Galdran ${ }^{a, b, *}$, David Pardo ${ }^{b, c, d}$, Artzai Picón ${ }^{a}$, Aitor Álvarez-Gila ${ }^{a}$ \\ ${ }^{a}$ TECNALIA Research E Innovation, Parque Científico y Tecnológico, Edificio 202, E-48170, Zamudio, Spain. \\ ${ }^{b}$ Department of Applied Mathematics, Statistics, and Operational Research, University of the Basque Country, Barrio Sarriena S/N, Campus de \\ Leioa, E-48940, Leioa, Spain. \\ ${ }^{c}$ Basque Center for Applied Mathematics, BCAM Bilbao, Spain. \\ ${ }^{d}$ IKERBASQUE, Basque Foundation for Science, Alameda Urquijo, 36-5, E-48011, Bilbao, Spain.
}

\begin{abstract}
Underwater images typically exhibit color distortion and low contrast as a result of the exponential decay that light suffers as it travels. Moreover, colors associated to different wavelengths have different attenuation rates, being the red wavelength the one that attenuates the fastest. To restore underwater images, we propose a Red Channel method, where colors associated to short wavelengths are recovered, as expected for underwater images, leading to a recovery of the lost contrast. The Red Channel method can be interpreted as a variant of the Dark Channel method used for images degraded by the atmosphere when exposed to haze. Experimental results show that our technique handles gracefully artificially illuminated areas, and achieves a natural color correction and superior or equivalent visibility improvement when compared to other state-of-the-art methods.
\end{abstract}

Keywords: Underwater image degradation, color correction, image dehazing, contrast enhancement, visibility recovery.

\section{Introduction}

The field of underwater imaging has drawn much attention over the last years. The possibility of improving underwater images by just using image processing techniques is very appealing due to the low cost of implementation when compared with more sophisticated techniques. These methodologies enjoy a wide range of applications, from marine biology and archaeology (1) to ecological research (2). The improvement of unmanned vehicles (ROV) navigation capabilities (3) is also a very important field of application. For example, underwater vehicle navigation can be guided through video mosaics (4). Mosaicing requires a previous registration of different input images (5), and image processing techniques are usually implemented as a preprocessing step to improve the results.

The problem of restoring images degraded by an underwater environment is challenging, in part because light traveling underwater suffers from two combined degradations, known as scattering and absorption. The former consists on a change of direction of light after collision with particles, and the latter explains how particles absorb light. The main consequences of these degradation processes are a decrease in the visibility distance and a color distortion that depends on the wavelength of each light beam. The shorter wavelengths (green and blue colors), reach further depths in the scene than the rapidly vanishing longest ones (red), transferring to the final image a characteristic bluishgreenish tone. Together with turbidity of the water, and the organic particles suspended on the medium, this yields a hard restoration problem, since overall performance of algorithms becomes highly dependent on environmental conditions. Often, an artificial light source is added to the imaging device to try to increment the visibility range in the scene, but this entails some disadvantages: the amount of power needed to illuminate underwater scenarios can be

\footnotetext{
${ }^{*}$ Corresponding author

Email address: adrian.galdran@tecnalia.com (Adrian Galdran ${ }^{a, b, *}$, David Pardo $^{b, c, d}$, Artzai Picón $^{a}$, Aitor Álvarez-Gila $^{a}$ )
} 
prohibitively expensive; moreover, illumination is not obtained in a global uniform way and a bright area in the center of the image tends to appear, as backscattering occurs from nearby particles causing visual disturbance.

The restoration of images degraded by an underwater environment with image processing techniques has been studied in various ways. For instance, in $(6,7)$ authors suggest an algorithm that takes as input two images obtained through a polarizer that is rotated to work at different orientations, obtaining in this way extra information about the scene that facilitates the inversion of the image formation process. Also, a supervised algorithm is presented in (8), where parameters of color correction are learnt over training data. The above methods require additional information, and do not work on single images without extra input. Single-image approaches can be found in (9), where they employ an image fusion strategy, or (10) and (11), where authors are more focused on color treatment. A more exhaustive review of these and other works can be found in the recent survey (12).

Underwater image restoration can also be considered as a distance-dependent degradation problem. In the last years, this field has experienced a remarkable progress, mainly in context of correction of atmospheric degradation, also known as dehazing. A key contribution was made by Koschmieder (13), where the following degradation model was introduced:

$$
\overrightarrow{\mathrm{I}}(x)=\overrightarrow{\mathrm{J}}(x) \mathrm{t}(x)+\overrightarrow{\mathrm{A}}(1-\mathrm{t}(x))
$$

was proposed. In $\sqrt{1}, \overrightarrow{\mathrm{I}}$ is the observed intensity, the input degraded color image, $\mathrm{t}$ is the transmission, which describes the amount of light that is not scattered nor absorbed, and reaches the observer (inversely related to the depth in the scene), $\overrightarrow{\mathrm{A}}$ is the airlight, physically related to the color of the haze, and $\overrightarrow{\mathrm{J}}$ is the scene radiance, which is associated to the non-degraded image.

Eq. (1) makes clear that information of the depth in the scene is encoded in the way color degrades with respect to distance. Different image processing and computer vision techniques have been developed in the last years, based on this fact. For example in (14), and more recently in (15), the authors infer depth information for retrieving the 3D structure of a scene, using two images captured in different conditions of visibility. However, one of the potential applications of this model is the possibility of restoring and enhancing images that suffer a degradation that is heavier as the depth in the scene increases. Many authors have built on Eq. (1) to develop new algorithms, e.g. (16), where the problem was approached through a variational formulation. However, one of the most popular methods up to date has been the Dark Channel Method, proposed in (17). The main idea is that one can directly solve for the scene radiance $\vec{J}$ in Eq. 11 provided that we are able to estimate airlight and transmission. To estimate these quantities, authors assume that a haze-free outdoor image is colorful, with shadows and textures everywhere, so locally at least one color channel has some pixel with low intensity (this assumption is called the Dark Channel Prior). This is not the case of the hazy input image, where every color channel has an added amount of haze, and the deeper in the scene, the more haze is added - this can be identified with the additive part of Eq. (1). This physical observation allows to estimate the depth in the scene and the airlight, which is extracted from a pixel in the horizon of the scene, see (17) for further details.

Unfortunately, the nature of the degradation induced by a marine environment prevents from directly applying the Dark Channel method. Absorption, which is not present in the atmosphere, since it is considered to be transparent, influences the way in which colors are lost in an underwater scene. Red channel rapidly loses intensity, while the green and blue ones keep their intensity longer. In this situation, the Dark Channel Prior has no sense anymore: being the image degraded or not, there is almost always one color channel with low intensity (the red one).

Several authors have proposed various modifications of the Dark Channel Method method to solve model (1) adapted to the underwater environment. In (18), authors modify the algorithm prior to reflect the difference in attenuation between colors, while in (19) they keep the algorithm essentially untouched and prefer to modify the model by adding some parameters reflecting the disparity of degradation in different wavelengths and depths. Unfortunately, while in (18) the different rates of color degradations are not taken into account, the method in (19) strongly depends on the choice of the parameters that govern the algorithm, limiting the capability of both approaches.

In this work, we propose a Red Channel method suitable for underwater images, which can be interpreted as a variant of the Dark Channel method employed in dehazing of atmospheric images. Our method is simple and robust, and it recovers part of the lost visibility range while correcting the color distortion produced by absorption. Additionally, it has fewer free parameters than previously existing methods, and can cope efficiently with artificial light sources possibly present in the scene. 


\section{Red Channel Underwater Image Restoration}

Examining in detail Eq. 11, we observe that the scene radiance $\overrightarrow{\mathrm{J}}(x)$ first goes through a multiplicative distortion and then through an additive distortion. According to the Beer-Lambert law, transmission $t$ is exponentially decreasing with respect to the distance.

For the case of haze in the atmosphere, the transmission of an object in the scene depends both on the composition of the portion of air that the light beam has traversed to reach it, and the distance from the observer. A useful simplification, first introduced in (13), is to assume that the atmosphere is homogeneous, which allows us to write:

$$
\mathrm{t}(x)=e^{-\beta d(x)},
$$

where $d(x)$ is the scene depth and $\beta$ is the (constant) attenuation coefficient. From (2), we see that at zero depth, the transmission becomes $\mathrm{t}(x)=1$, and airlight attenuation has no influence in Eq. $(1$, which reduces to $\overrightarrow{\mathrm{I}}(x)=\overrightarrow{\mathrm{J}}(x)$. Conversely, as the depth in the scene increases, transmission decreases to zero, making the airlight contribution in (1) exponentially more significative, until it dominates the whole scene, effectively modeling what happens in the horizon of natural hazy scenes, $\overrightarrow{\mathrm{I}}(x) \approx \overrightarrow{\mathrm{A}}$. All the above intuition can be translated to the underwater environment with some adaptations. To that end, we rearrange the original model (1) and write:

$$
\begin{aligned}
\mathbf{1}-\mathrm{I}^{R} & =\mathrm{t}\left(1-\mathrm{J}^{R}\right)+(\mathbf{1}-\mathrm{t})\left(\mathbf{1}-\mathrm{A}^{R}\right) \\
\mathrm{I}^{G} & =\mathrm{tJ}^{G}+(\mathbf{1}-\mathrm{t}) \mathrm{A}^{G} \\
\mathrm{I}^{B} & =\mathrm{tJ}^{B}+(1-\mathrm{t}) \mathrm{A}^{B},
\end{aligned}
$$

being $\overrightarrow{\mathrm{I}}=\left(\mathrm{I}^{R}, \mathrm{I}^{G}, \mathrm{I}^{B}\right)$ and $\overrightarrow{\mathrm{J}}=\left(\mathrm{J}^{R}, \mathrm{~J}^{G}, \mathrm{~J}^{B}\right)$ the degraded and the original image respectively. Notice that Eqs. 3 are completely equivalent to Eq. (11). Therefore, it still reflects the fact that the light attenuates with distance, as it actually happens underwater. The main difference we must have into account is that red intensity decays faster as distance increases. To reflect this fact, we suggest the following modification of the Dark Channel prior, which we denote as Red Channel Prior. It states that:

$$
\mathrm{J}^{\mathrm{RED}}(x):=\min \left(\min _{y \in \Omega(x)}\left(\mathbf{1}-\mathrm{J}^{R}(y)\right), \min _{y \in \Omega(x)}\left(\mathrm{J}^{G}(y)\right), \min _{y \in \Omega(x)}\left(\mathrm{J}^{B}(y)\right)\right) \approx 0,
$$

for a non-degraded underwater image. Here, $\Omega(x)$ is a neighborhood of pixels around the $x$ location. Notice that for a degraded image near the observer, the red channel still keeps some intensity, so its reciprocal $1-\mathrm{J}^{R}$ is low, and the prior is still true. However, as distance increases, red intensity rapidly decays, and its weight in the Red Channel image decreases, so the prior begins to be false. This fact will allow us to estimate the depth in the scene, as well as the color of the water veil.

Remark 2.1. Notice that we denote the Red Channel of an image $\overrightarrow{\mathrm{J}}=\left(\mathrm{J}^{R}, \mathrm{~J}^{G}, \mathrm{~J}^{B}\right)$, given by Eq. (4), as $\mathrm{J}^{\mathrm{RED}}$, while its red component is denoted by $\mathrm{J}^{R}$.

\subsection{WaterLight estimation from Red Channel}

The first thing we need to estimate is the color of the water. We ideally want to pick a pixel that lies at the maximum depth with respect to the camera. Since we are assuming that the degradation is distance dependent, this location naturally corresponds to the maximum values in the Red Channel of the original image.

Let us denote $\overrightarrow{\mathrm{A}}=\overrightarrow{\mathrm{I}}\left(x_{0}\right)=\left(1-\mathrm{A}^{R}, \mathrm{~A}^{G}, \mathrm{~A}^{B}\right)$. We choose as the waterlight $x_{0}$ the pixel in the degraded image that corresponds to the brightest pixel in its Red Channel:

$$
\overrightarrow{\mathrm{A}}=\left(\mathrm{I}^{R}\left(x_{0}\right), \mathrm{I}^{G}\left(x_{0}\right), \mathrm{I}^{B}\left(x_{0}\right)\right) \text { such that } \mathrm{I}^{\mathrm{RED}}\left(x_{0}\right) \geq \mathrm{I}^{\mathrm{RED}}(x) \quad \forall x .
$$

The analogous method to the one suggested in (17) would be to take the top $10 \%$ brightest pixels in the Red Channel image, and among this set of pixels, choose the one that is brightest in the degraded image. This pixel does not need to be the brightest pixel in the Red Channel. However, the brightest pixel in the degraded image is an ambiguous definition. In the underwater case, we have observed that picking among that $10 \%$ of pixels the one that has lower red component obtains the best results in our experiments, see Fig. 1 . 


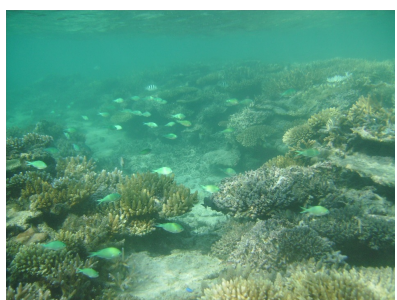

(a)

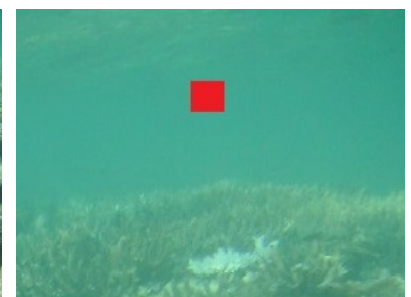

(b)

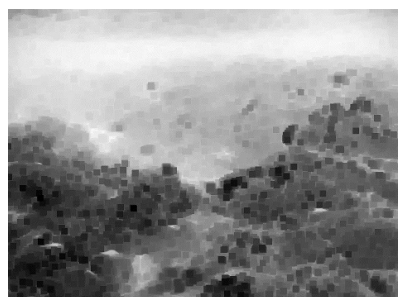

(c)

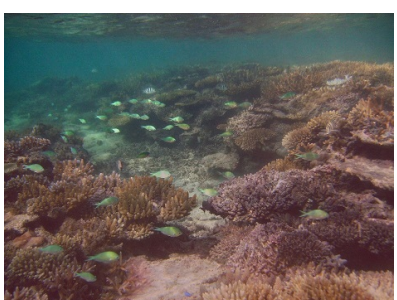

(d)

Figure 1: Waterlight detection from Red channel. (a) Original image, the red rectangle marks the region from where waterlight was extracted (b) In red, exact pixel location of waterlight within the rectangle marked in (a) (c) Red Channel of the original image (d) Final restoration result

\subsection{Transmission Estimate from Red Channel}

After estimating the waterlight, we are now able to produce an estimate for the transmission of the scene. This is achieved with the following result:

Theorem 2.2. Given an estimate $\overrightarrow{\mathrm{A}}=\left(\mathrm{A}^{R}, \mathrm{~A}^{G}, \mathrm{~A}^{B}\right)$ for the waterlight, assuming that transmission is locally constant, and under the Red Channel hypothesis (4), $\mathrm{t}(x)$ can be estimated as:

$$
\widetilde{\mathrm{t}}(x)=\mathbf{1}-\min \left(\frac{\min _{y \in \Omega(x)}\left(\mathbf{1}-\mathrm{I}^{R}(y)\right)}{\mathbf{1}-\mathrm{A}^{R}}, \frac{\min _{y \in \Omega(x)} \mathrm{I}^{G}(y)}{\mathrm{A}^{G}}, \frac{\min _{y \in \Omega(x)} \mathrm{I}^{B}(y)}{\mathrm{A}^{B}}\right) .
$$

Proof: It is a simple adaptation of the steps of the derivation in (17). For details, see the Appendix.

Let us stress that model 3 is in fact a convex combination with coefficients $t$ and $1-t$, so the estimate $\tilde{t}$ of $t$ must also lie in $[0,1]$. This is a non-trivial fact that relies on how we performed the estimation of the waterlight. We have:

Theorem 2.3. Transmission estimate (5) verifies $\widetilde{\mathrm{t}}(x) \in[0,1]$ for all pixel $x$ in the image.

Proof: See the Appendix.

Nevertheless, this straightforward extension of the Dark Channel Method to include the Red Channel Prior fails to take into account the velocity of different wavelengths in the light. To incorporate this behavior in our model, we suggest two possibilities. The first one is performed in the transmission estimate step, and consists of extending the model (3) to involve three transmission maps, one for each color component. The second one occurs during the final inversion step, and consists of weighting the additive contribution of each component of the waterlight.

\section{Incorporating Color Correction I: Vectorial Transmission}

In our work, we select the former approach. Departing from (3) and distinguishing three different transmission maps, we have:

$$
\begin{aligned}
\mathbf{1}-\mathrm{I}^{R} & =\mathrm{t}^{R}\left(1-\mathrm{J}^{R}\right)+\left(\mathbf{1}-\mathrm{t}^{R}\right)\left(\mathbf{1}-\mathrm{A}^{R}\right) \\
\mathrm{I}^{G} & =\mathrm{t}^{G} \mathrm{~J}^{G}+\left(\mathbf{1}-\mathrm{t}^{G}\right) \mathrm{A}^{G} \\
\mathrm{I}^{B} & =\mathrm{t}^{B} \mathrm{~J}^{B}+\left(1-\mathrm{t}^{B}\right) \mathrm{A}^{B}
\end{aligned}
$$

In (6), we need to estimate the three components of the waterlight, and the three transmission maps, one for each color. However, these three matrixes are not independent: using (2), we conclude that we only need to estimate one matrix and two scalar numbers. We have:

$$
\begin{aligned}
& \mathrm{t}^{R}(x)=e^{-\beta^{R} d(x)} \\
& \mathrm{t}^{G}(x)=e^{-\beta^{G} d(x)}=\left(e^{-\beta^{R} d(x)}\right)^{\frac{\beta^{G}}{\beta^{R}}}=\left(\mathrm{t}^{R}(x)\right)^{\lambda_{G}} \\
& \mathrm{t}^{B}(x)=e^{-\beta^{B} d(x)}=\left(e^{-\beta^{R} d(x)}\right)^{\frac{\beta^{B}}{\beta^{R}}}=\left(\mathrm{t}^{R}(x)\right)^{\lambda_{B}},
\end{aligned}
$$


where $\lambda_{G}=\beta^{G} / \beta^{R}$ and $\lambda_{B}=\beta^{B} / \beta^{R}$. This leads to a slightly more difficult (but still possible) estimation of the transmission, by modification of the Red Channel prior. We can check the following property:

Theorem 2.4. Assuming that we have already estimated the waterlight, and the attenuation coefficients for the green and blue transmission maps, $\lambda_{G}$ and $\lambda_{B}$, and that:

$$
\begin{gathered}
\min \left(\min _{y \in \Omega(x)}\left(\mathbf{1}-\mathrm{J}^{R}(y)\right) /\left(\mathbf{1}-\mathrm{A}^{R}\right), \mathrm{t}_{R}^{\lambda_{G}-1}(x)\left(\min _{y \in \Omega(x)} \mathrm{J}^{G}(y)\right) / \mathrm{A}^{G}+\right. \\
\left.\mathbf{1}-\mathrm{t}_{R}^{\lambda_{G}-1}(x), \mathrm{t}_{R}^{\lambda_{B}-1}(x)\left(\min _{y \in \Omega(x)} \mathrm{J}^{B}(y)\right) / \mathrm{A}^{B}+\mathbf{1}-\mathrm{t}_{R}^{\lambda_{B}-1}(x)\right) \equiv 0,
\end{gathered}
$$

the red, green, and blue components of the transmission map can be estimated as:

$$
\begin{aligned}
& \mathrm{t}^{R}(x)=\mathbf{1}-\min \left(\frac{\min _{y \in \Omega(x)} \mathbf{1}-\mathrm{I}^{R}(y)}{\mathbf{1}-\mathrm{A}^{R}}, \frac{\min _{y \in \Omega(x)} \mathrm{I}^{G}(y)}{\mathrm{A}^{G}}, \frac{\min _{y \in \Omega(x)} \mathrm{I}^{B}(y)}{\mathrm{A}^{B}}\right) \\
& \left.\widetilde{\mathrm{t}}^{G}(x)=\widetilde{(\mathfrak{t}}^{R}(x)\right)^{\lambda_{G}} \\
& \widetilde{\mathrm{t}}^{B}(x)={\left.\widetilde{\left(\mathrm{t}^{R}\right.}(x)\right)^{\lambda_{B}}}
\end{aligned}
$$

Proof: Details are given in the Appendix.

Remark 2.5. Notice that in formula (8) there are two convex combinations,

$$
\begin{aligned}
& \mathrm{t}_{R}^{\lambda_{G}-1}\left(\min _{\Omega} \mathrm{J}^{G} / \mathrm{A}^{G}\right)+\left(\mathbf{1}-\mathrm{t}_{R}^{\lambda_{G}-1}\right) \mathbf{1} \\
& \mathrm{t}_{R}^{\lambda_{B}-1}\left(\min _{\Omega} \mathrm{J}^{B} / \mathrm{A}^{B}\right)+\left(\mathbf{1}-\mathrm{t}_{R}^{\lambda_{B}-1}\right) \mathbf{1},
\end{aligned}
$$

where these quantities vary from $\min _{\Omega} \mathrm{J}^{G} / \mathrm{A}^{G}$ and $\min _{\Omega} \mathrm{J}^{B} / \mathrm{A}^{B}$ respectively, to 1 . Let us analyze the extreme cases of this generalized prior.

- When these two quantities are equal to 1 , then $\mathrm{t}_{R}^{\lambda_{G}-1}=\mathrm{t}_{R}^{\lambda_{G}} / \mathrm{t}_{R}=0$, and $\mathrm{t}_{R}^{\lambda_{B}-1}=\mathrm{t}_{R}^{\lambda_{B}} / \mathrm{t}_{R}=0$, implying that the transmission is null, so the depth is maximum. In this case, assumption (8) reduces to the red channel prior (4), so we are enforcing our main hypothesis in regions of high depth in the scene.

- When they are $\min _{\Omega} \mathrm{J}^{G} / \mathrm{A}^{G}$ and $\min _{\Omega} \mathrm{J}^{B} / \mathrm{A}^{B}$ respectively, then $\mathrm{t}_{R}^{\lambda_{G}-1}=\mathrm{t}_{R}^{\lambda_{G}} / \mathrm{t}_{R}=1$ and $\mathrm{t}_{R}^{\lambda_{B}-1}=\mathrm{t}_{R}^{\lambda_{B}} / \mathrm{t}_{R}=1$, so $\mathrm{t}_{R}=\mathrm{t}_{G}=\mathrm{t}_{B}$. This situation can only happen when we are near the observer, where the degradation is minimum, and more intense red can be found, so $\left(\min _{y \in \Omega(x)} \mathbf{1}-\mathrm{J}^{R}(y)\right) /\left(\mathbf{1}-\mathrm{A}^{R}\right)$ is near zero, making hypothesis $(8)$ valid.

The fact that the three derived transmission maps still lie in the interval $[0,1]$ follows directly from Theorem 2.3

Remark 2.6 (Transmission Map Refinement). In all these calculations, we assume that the transmission map can be considered patch-wise constant, but this is not realistic. If the patch contains a depth jump, this hypothesis fails and leads to artifacts in the recovered scene. A solution is to refine the calculated rough transmission map using the guided filter (20), a version of the bilateral filter that efficiently captures the fine details of the degraded image, and incorporates them in the estimated transmission map, see Fig. 2. This method is faster and has no noticeable loss of detail with respect to the method based on the image matting technique (21), originally used in (17). 

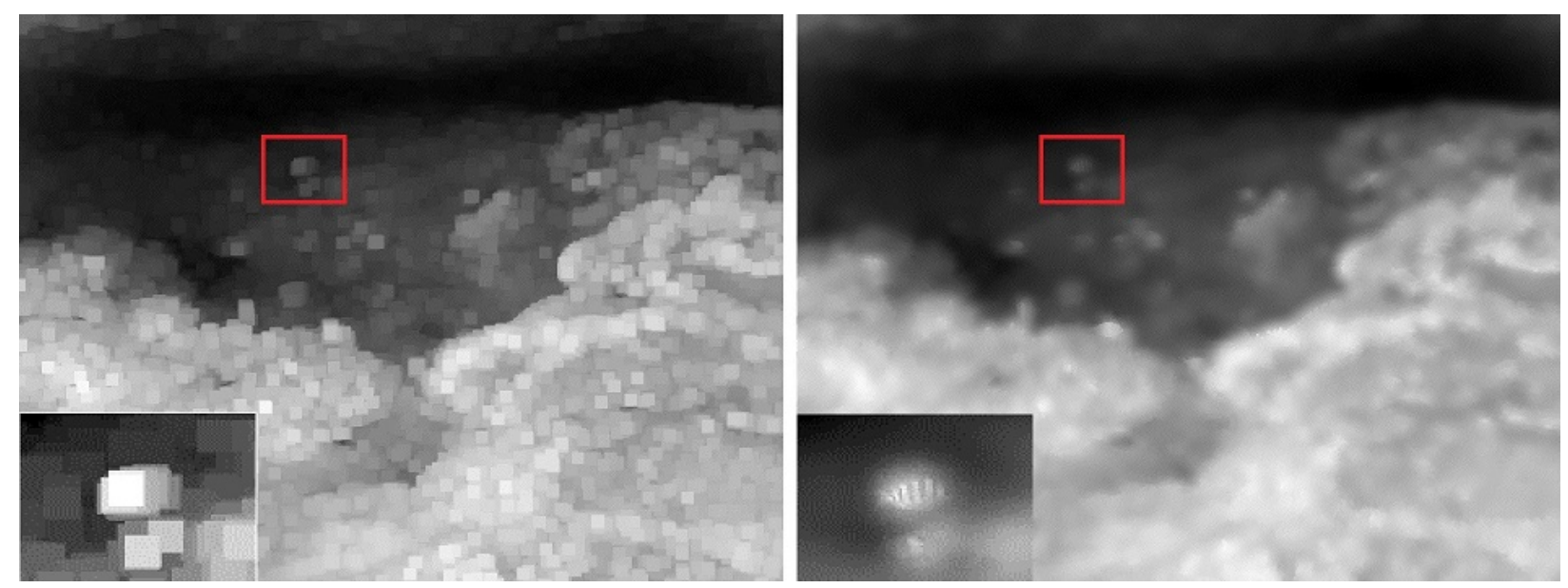

Figure 2: Transmission map refinement

\subsection{Final Inversion}

To solve (1), we need to invert channelwise Eqs. (3). We prevent dividing by small numbers by putting a lower bound on the denominator:

$$
\begin{aligned}
& \mathrm{J}^{R}(x)=\frac{\mathrm{I}^{R}(x)-\mathrm{A}^{R}}{\max \left(\mathrm{t}^{R}(x), \mathrm{t}_{0}\right)}+\mathrm{A}^{R} \\
& \mathrm{~J}^{G}(x)=\frac{\mathrm{I}^{G}(x)-\mathrm{A}^{G}}{\max \left(\mathrm{t}^{G}(x), \mathrm{t}_{0}\right)}+\mathrm{A}^{G} \\
& \mathrm{~J}^{B}(x)=\frac{\mathrm{I}^{B}(x)-\mathrm{A}^{B}}{\max \left(\mathrm{t}^{B}(x), \mathrm{t}_{0}\right)}+\mathrm{A}^{B}
\end{aligned}
$$

where a typical value for $\mathrm{t}_{0}$ can be 0.1 .

\section{Incorporating Color Correction II: Weighted WaterLight}

Attenuation coefficients $\lambda_{G}$ and $\lambda_{B}$ in 99 are not easy to determine, as they depend on the type of water. They are usually specified heuristically or left as free parameters. In our case, a simplified version of our technique can be obtained by directly building $t$ as in (5) and letting the additive waterlight weight the contributions of each wavelength. This yields a slightly different inversion formula:

$$
\begin{aligned}
& \mathrm{J}^{R}(x)=\frac{\mathrm{I}^{R}(x)-\mathrm{A}^{R}}{\max \left(\mathrm{t}(x), \mathrm{t}_{0}\right)}+\rho^{R} \mathrm{~A}^{R}, \\
& \mathrm{~J}^{G}(x)=\frac{\mathrm{I}^{G}(x)-\mathrm{A}^{G}}{\max \left(\mathrm{t}(x), \mathrm{t}_{0}\right)}+\rho^{G} \mathrm{~A}^{G}, \\
& \mathrm{~J}^{B}(x)=\frac{\mathrm{I}^{B}(x)-\mathrm{A}^{B}}{\max \left(\mathrm{t}(x), \mathrm{t}_{0}\right)}+\rho^{G} \mathrm{~A}^{B},
\end{aligned}
$$

where parameters $\rho^{R}, \rho^{G}, \rho^{B}$ weight the contribution of each component. The multiplicative part involving $\overrightarrow{\mathrm{I}}$ takes care of the restoration of the deep parts of the scene, while the additive part removes the color cast. Our experience shows that there is no need to estimate these coefficients, since the reciprocal of the waterlight coefficients can automatically fulfil this task. Thus, a final version of our algorithm would include the following inversion formula:

$$
\mathrm{J}^{\alpha}(x)=\frac{\mathrm{I}^{\alpha}(x)-\mathrm{A}^{\alpha}}{\max \left(\mathrm{t}(x), \mathrm{t}_{0}\right)}+\left(1-\mathrm{A}^{\alpha}\right) \mathrm{A}^{\alpha},
$$

for $\alpha \in\{R, G, B\}$. This implementation no longer guarantees that the resultant image lies in $[0,1]$. However, a simple min-max normalization of the intensity values to carry them to the unit interval easily fixes this issue. 

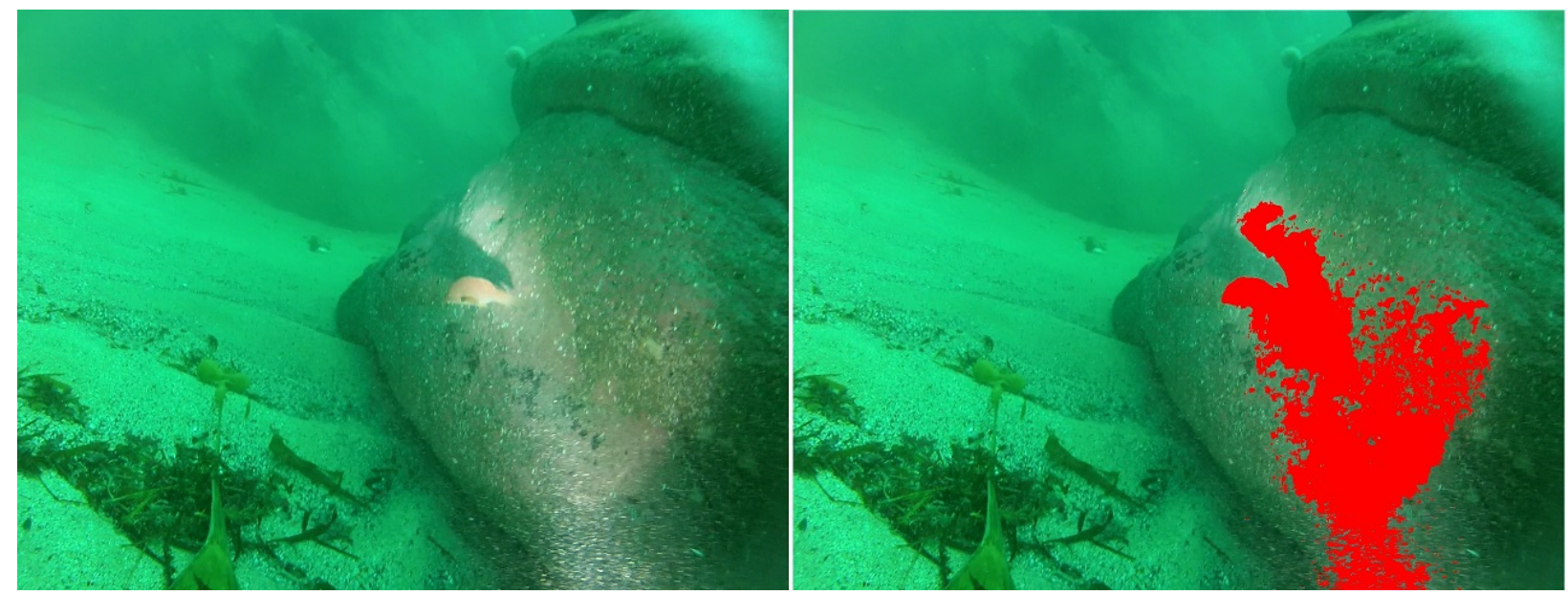

Figure 3: Saturation-based segmentation of artificially illuminated areas. Pixels with $S(x)<0.4$ are painted red.

\subsection{Handling Artificial Illumination}

Artificially illuminated underwater images are quite common. Unfortunately, high intensity of the pixels does not necessarily indicate the presence of artificial light. A much more adequate cue to characterize it is the saturation. The saturation component represents the purity of the chromaticity of a pixel: when a color is in the pure spectrum, it is completely saturated, containing no white light. A color loses saturation when we add white light, which contains power at all wavelengths (22). The lack of saturation can thus be interpreted as the amount of white light appearing within its color. Saturation is defined as:

$$
\operatorname{Sat}(\overrightarrow{\mathrm{I}})=\frac{\max \left(\mathrm{I}^{R}, \mathrm{I}^{G}, \mathrm{I}^{B}\right)-\min \left(\mathrm{I}^{R}, \mathrm{I}^{G}, \mathrm{I}^{B}\right)}{\max \left(\mathrm{I}^{R}, \mathrm{I}^{G}, \mathrm{I}^{B}\right)}
$$

In Fig. 3, a saturation-based segmentation effectively separates areas with artificial light from the rest of the image. Additionally, when no light is present in the scene, the saturation is notably different from zero. This reflects the fact that artificial illumination forces a pixel to have similar intensity values rather than more green/blue than red. Then, a low saturation indicates that $\max \left(\mathrm{I}^{R}, \mathrm{I}^{G}, \mathrm{I}^{B}\right) \simeq \min \left(\mathrm{I}^{R}, \mathrm{I}^{G}, \mathrm{I}^{B}\right)$, i.e., $\mathrm{I}^{R} \simeq \mathrm{I}^{G} \simeq \mathrm{I}^{B}$.

It is easy to incorporate saturation into our prior. We just extend (4) to the following Red-Saturation Prior:

$$
\begin{gathered}
\mathrm{J}^{\mathrm{RED}-\mathrm{SAT}}(x)=\min \left(\min _{y \in \Omega(x)}\left(\mathbf{1}-\mathrm{J}^{R}(y)\right), \min _{y \in \Omega(x)}\left(\mathrm{J}^{G}(y)\right),\right. \\
\left.\min _{y \in \Omega(x)}\left(\mathrm{J}^{B}(y)\right), \min _{y \in \Omega(x)} \operatorname{Sat}(y)\right) \equiv 0
\end{gathered}
$$

This extension handles illumination gracefully. If a pixel exhibits disparity in the three channels, it is because the red channel has already lost intensity, so the object is far away from the observer and needs to be restored. However, if a pixel has a value for the three channels that is far from zero, it lies in a location near the observer, or in an artificially illuminated area. Without adding saturation to (4), an artificially illuminated area that is not close to the observer can have intermediate values in the three channels, e.g. $(0.5,0.5,0.5)$. The Red Channel Prior considers this pixel to be far away, while (12) classifies it as artificially illuminated, and will not attempt to restore it, as desired.

To implement this idea within the Red Channel methodology, we simply reformulate Theorem 2.2 as:

Theorem 2.7. Under artificial illumination, we can compute an improved estimate of $\mathrm{t}(x)$ as:

$$
\widetilde{\mathrm{t}}(x)=\mathbf{1}-\min \left(\frac{\min _{y \in \Omega(x)}\left(\mathbf{1}-\mathrm{I}^{R}(y)\right)}{\mathbf{1}-\mathrm{A}^{R}}, \frac{\min _{y \in \Omega(x)} \mathrm{I}^{G}(y)}{\mathrm{A}^{G}}, \frac{\min _{y \in \Omega(x)} \mathrm{I}^{B}(y)}{\mathrm{A}^{B}}, \lambda \min _{y \in \Omega(x)} \operatorname{Sat}(y)\right),
$$




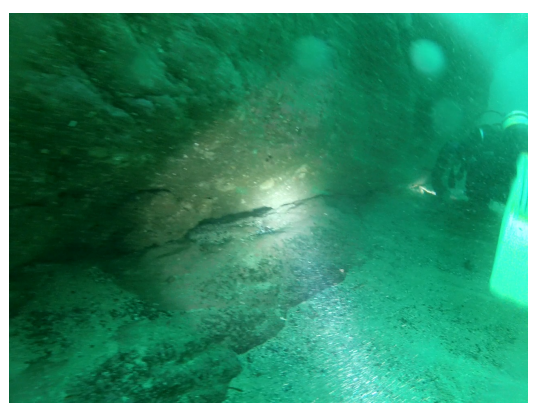

(a)

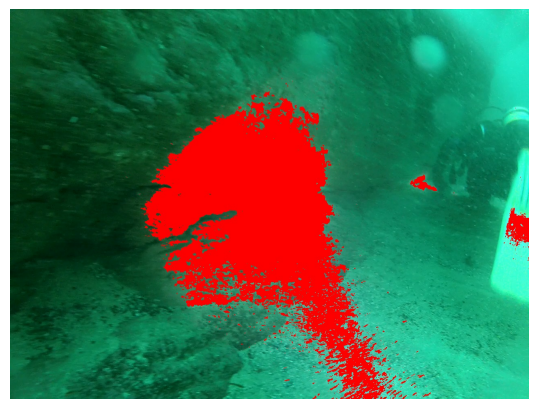

(d)

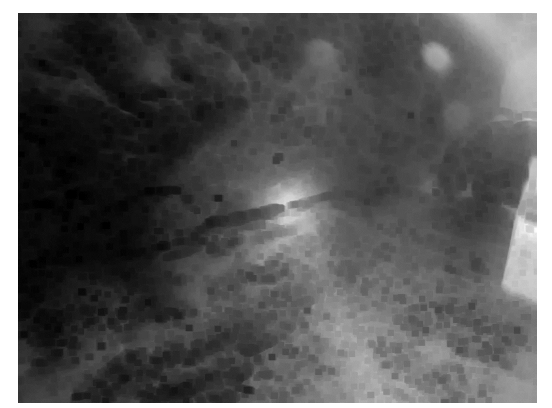

(b)

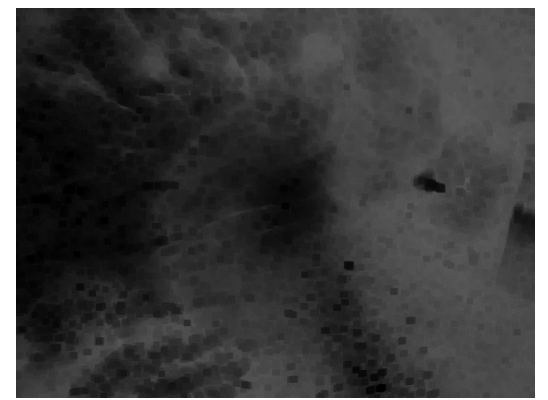

(e)

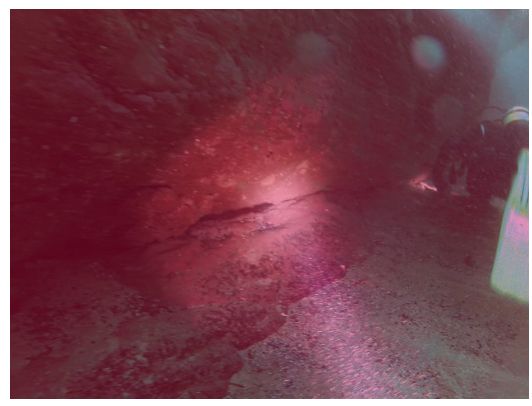

(c)

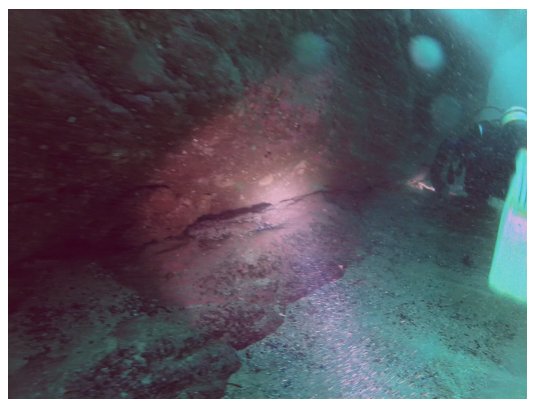

(f)

Figure 4: Result of considering or not the Saturation cue. (a) Image with artificial light source on it. (b) Red Channel obtained with Eq. 55. (c) Image after processing without saturation prior. (d) Saturation-based segmentation of artificially illuminated areas. (e) Improved Red-Saturation Channel obtained with Eq. A6. (f) Image after processing with saturation prior.

where $\lambda \in[0,1]$ is a scalar multiplier that can be manually adjusted to suit the amount of artificial light we want to take into account.

Proof: It is a simple extension of Theorem 2.2

The effect of including saturation can be clearly appreciated in Fig. 4 . Therein, Fig. (4a) displays an artificially illuminated scene. Fig. (4b) shows the Red Channel calculated as in Eq. (5), where artificially illuminated pixels are classified as if they were in the background of the scene. In Fig. (4d) we see the segmentation of illuminated areas, while Fig. 4e shows the Red-Saturation Channel, computed using Eq. 12]. In this figure, misclassified pixels are now darker, implying that they are closer to the observer, and do not need to be restored. Figs. (4c) and (4f) show the results of restoration with and without the Red-Saturation Prior respectively. We can appreciate a chromatic artifact that renders the restored image in a reddish tone at illuminated areas, due to a wrong depth estimation. The integration of Saturation in the transmission estimation considerably reduces this effect, as seen in Fig. (4f).

\section{Results}

It is very hard to assess the performance of an underwater image restoration algorithm, since there is not groundtruth available. Hence, no standard non-referenced image quality metric, such as PSNR or SSIM, can be used in our situation. In this work, we evaluate the restoration output in a twofold manner. First, we will assess the amount of visibility that a our algorithm, together with other five state-of-the-art methods, is able to recover. Second, we propose to evaluate color correction based on the examination of three indicators of the quality of the restoration, that will be described in the next section. Each of these indicators reveals the weaknesses and strengths of the methods we compare in a different aspect of color quality.

For comparison purposes, we have selected the four test images in Fig. 5, since they have been captured under different conditions, with a varied type of waters, representing different scene configurations and with a varying degree of artificial illumination. 


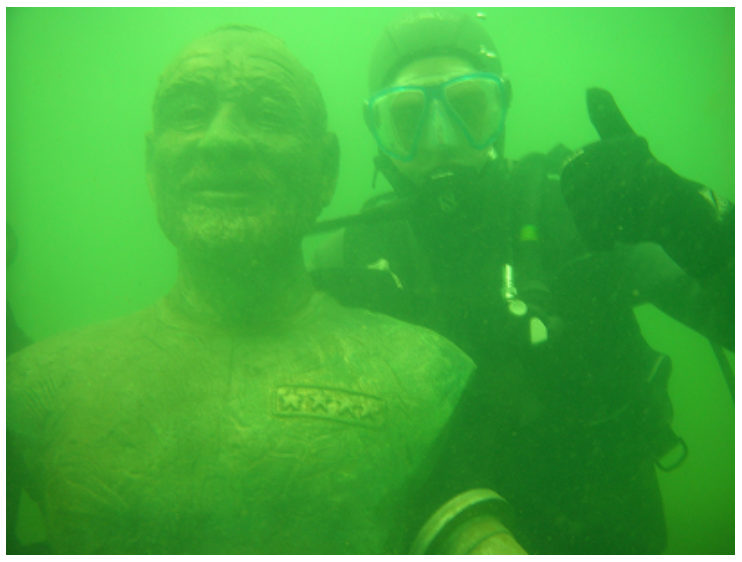

(a)

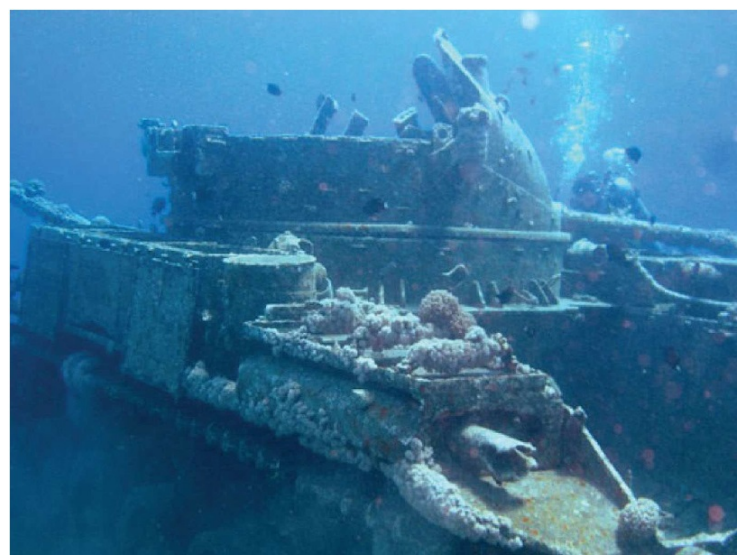

(c)

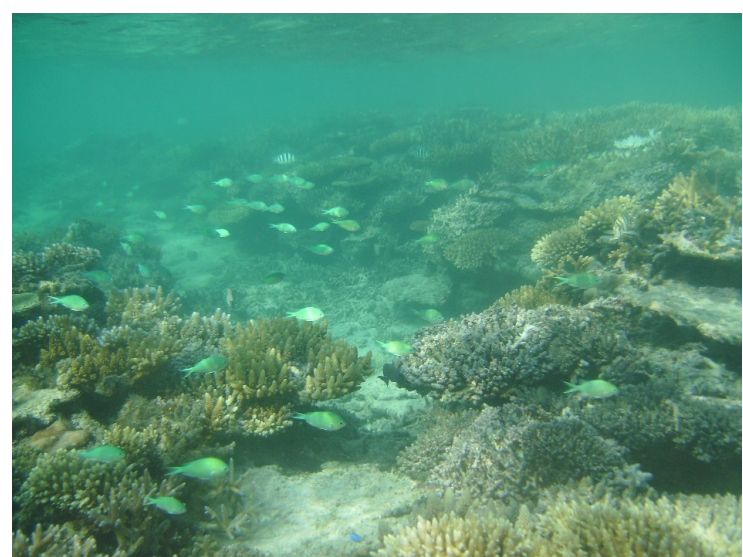

(b)

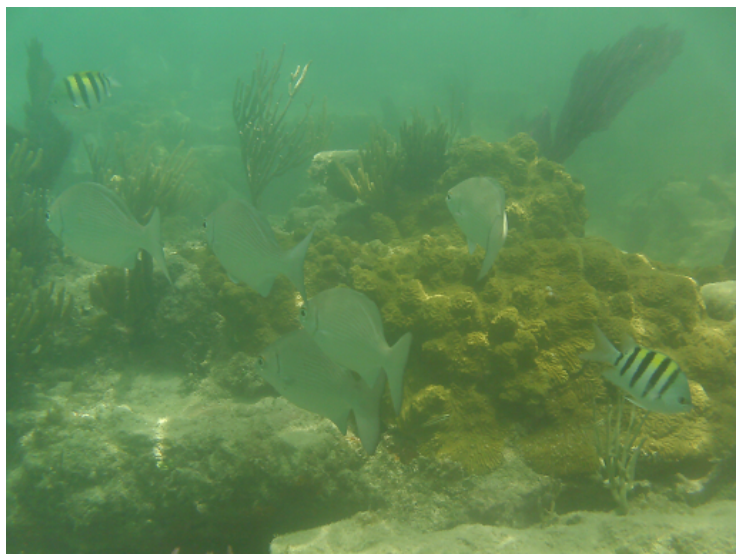

(d)

Figure 5: The set of images chosen for evaluation. a) Diver image b) Open scene image c) Shipwreck image e) Fishes image

\subsection{Contrast and Visibility Recovery}

In this work, to evaluate the amount of contrast recovered we rely on a non-referenced metric proposed in (23) and used in (24) for purposes of visibility recovery assessment, on images corrupted by fog. It is meaningful to use this metric, since it aims at finding whether the algorithm has retrieved some of the edges that were lost by the scattering effect. It works by computing three coefficients that measure the amount of new visible edges that a given contrast restoration method produces, as well as the quality of the restoration, since retrieving many edges alone is not an indicator of the quality of the algorithm.

We have compared extensively the performance of our algorithm with other five state-of-the-art techniques for underwater image restoration and enhancement by computing the coefficients $e$ and $r$, see (23). The first of these coefficients, $e$, is calculated by first building a map of visible edges on the restored images, and then, counting the amount of edges on the original image, $n_{r}$, and on the restored image, $n_{o}$, and setting:

$$
e=\frac{n_{r}-n_{o}}{n_{o}} .
$$

This evaluates the quantity of edges that were not present at $I_{o}$ but are in $I_{r}$. Complementarily, for each pixel $i$ belonging to a visible edge, it computes the ratio of the gradient in the restored image and in the original image $r_{i}$, 
Table 1: Visibility recovery coefficient $e$ compared on four images

\begin{tabular}{c|cccc}
\hline \hline e coefficient & Diver & Open Scene & Shipwreck & Fishes \\
\hline Ancuti et al. (9) & 23.7132 & 0.06377 & 1.4890 & 7.5038 \\
Malkasse et al. (25) & 2.4075 & 0.07222 & 0.5599 & 3.0220 \\
Carlevaris et al. (18) & 0.1617 & -0.2039 & 1.3534 & 0.5345 \\
Chiang et al. (19) & 0.1046 & 0.1705 & 0.3123 & 0.1842 \\
Serikawa et al. (26) & 0.4414 & 0.14903 & 0.0381 & 0.9163 \\
Ours & 16.4695 & 0.4011 & 1.8982 & 4.5547
\end{tabular}

Table 2: Restoration quality coefficient $r$ compared on four images

\begin{tabular}{c|cccc}
\hline \hline r coefficient & Diver & Open Scene & Shipwreck & Fishes \\
\hline Ancuti et al. (9) & 4.7713 & 1.7827 & 1.4890 & 5.3905 \\
Malkasse et al. (25) & 5.9437 & 3.6533 & 4.2686 & 6.3280 \\
Carlevaris et al. (18) & 1.2083 & 1.3625 & 2.6018 & 0.9813 \\
Chiang et al. (19) & 1.2976 & 1.7812 & 1.7677 & 1.4304 \\
Serikawa et al. (26) & 1.5719 & 2.1414 & 2.0557 & 1.8693 \\
Ours & 2.1197 & 1.1235 & 1.9810 & 2.3799
\end{tabular}

and geometrically averages it to obtain the coefficient $r$ as:

$$
r=\frac{1}{n_{r}} \sum_{i} \log \left(r_{i}\right) .
$$

Finally, it also computes the number $n_{s}$ of pixels that the algorithm saturates to black or white, normalized by the size of the image, obtaining the third coefficient $\sigma$ :

$$
\sigma=\frac{n_{s}}{\operatorname{dim}_{x} \times \operatorname{dim}_{y}}
$$

The visual results of restoring these images with the algorithms of $(9 ; 25 ; 18,19 ; 26)$ and ours are shown in Figs. (6), (8), (10), (12), and the edge maps computed by the evaluation metric we are using are given in (7), (9), (11), (13). Tables 1 and 2 show the $e$ and $r$ coefficients that the different methods scored in these set of images. The $\sigma$ coefficient is not displayed, since all methods exhibit values very close to zero.

We see that only on the Diver and Fishes images, there is one method, Ancuti's algorithm, that outperforms our results. However, in Table 2 we can see that Ancuti's method is producing a higher $r$ value. That means that this method is recovering spurious edges, as confirmed by visual inspection of Figs. (7a) and (7f), as well as of (9a) and (9f). We see there how Ancuti's algorithm is recovering much more noise that our method on areas of the image that contain no object, only water.

In every other case, our proposed algorithm performs better than the other state-of-the-art methodologies in terms of visibility recovery, analyzed through the $e$ coefficient. The values of the $r$ coefficient in our method tend to be higher than Carlevaris, Chiang's and Serikawa's, but this is due to the fact that these three methods are not recovering as much visibility as ours, as reflected by their scores at the $e$ coefficient table. Since they retrieve substantially less edges, they also recover less spurious edges. 


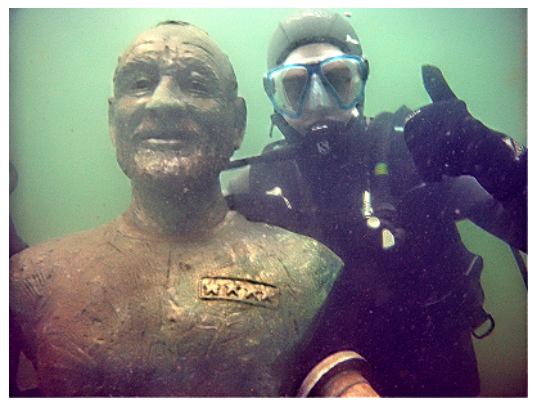

(a)

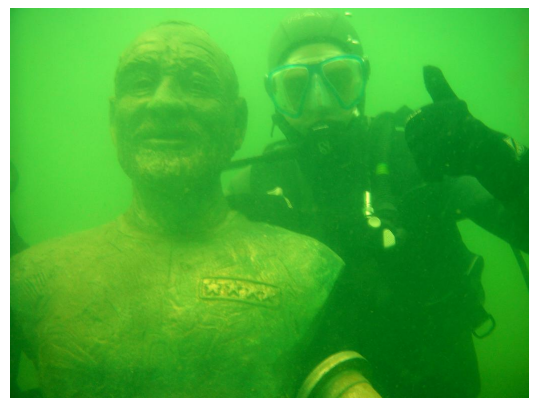

(d)

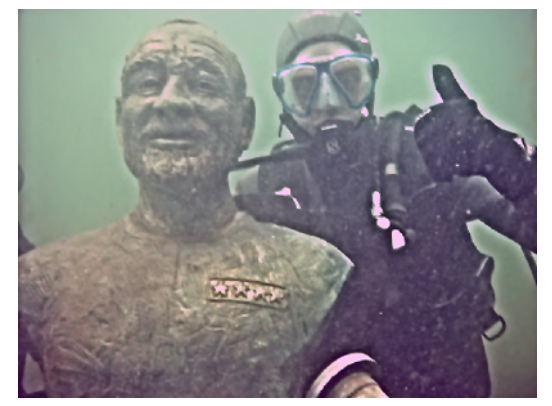

(b)

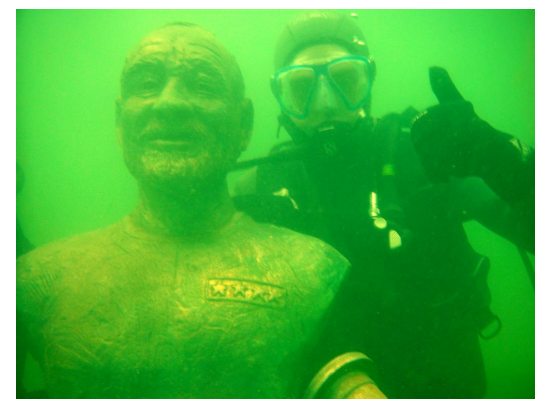

(e)

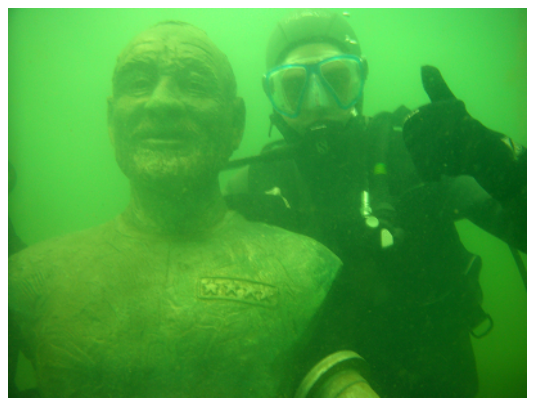

(c)

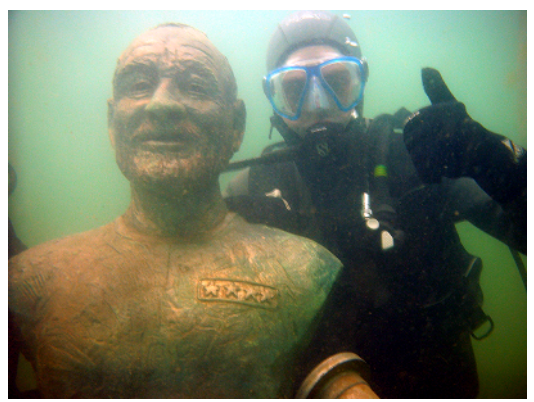

(f)

Figure 6: Results on the Diver image. (a) With (9) (b) With (25) (c) With (18) (d) With (19) (e) With (26) (f) With the Red Channel algorithm.

\subsection{Color Correction Evaluation}

In terms of color correction, as there is no groundtruth of how should the color on the scene really be, we can only evaluate the ability of each method to remove the color cast and recover a natural color distribution. Since naturalness of colors is a rather subjective concept, it is very hard to measure the quality of the recovered colors.

Other works, see for instance $(18,19 ; 27)$, try to assess the ability of a method to recover realistic colors by taking an image of a color board before and after immersion in a water body, then processing the water-degraded image and comparing the result to the colors of the image acquired in the atmosphere. This can be a meaningful evaluation approach for a single method performance analysis. Unfortunately, this approach is little suitable for comparison tasks, as it is very particular, and hardly reproducible, since each immersion takes place in different waters and under very different conditions that could favor one or another method. Moreover, it is arguable whether an underwater image processing algorithm should have the goal to convert colors to their equivalent in the surface. Our goal here is to obtain an improved image, in terms of contrast and visibility increase. Regarding color correction, it must be performed in a way that the resultant image looks natural to the human eye, and this is inevitably a subjective task that does not necessarily match the idea of converting an underwater image to its surface counterpart.

For these reasons, we rather analyze the behavior of the different methods with respect to three visual components that are affected by color corruption, namely, color dominance, color cast and color fading which leads to low- color saturation. We propose one metric for each of these aspects, allowing to quantitatively evaluate the performance of each method in a way that proves to be consistent with subjective perceptual criteria when applied to the test images we handle here.

As advocated in (28), we can associate an image with color cast to the fact that one chromatic channel exhibits a particularly different standard deviation with respect to others. Moreover, color cast should not be confused with color dominance. In the latter case, it is the average value of a channel that predominates. This interpretation in fact further motivates the idea that contrast enhancement in color corrupted images can help to decrease color cast, as it spreads the intensity values of all color channels, decreasing thus the dispersion of the correspondent standard deviations.

We can thus set as a meaningful goal to reduce color cast, while not increasing color dominance on the three chromatic components of an underwater image. To measure the achievement of these goals, we look at the relative 


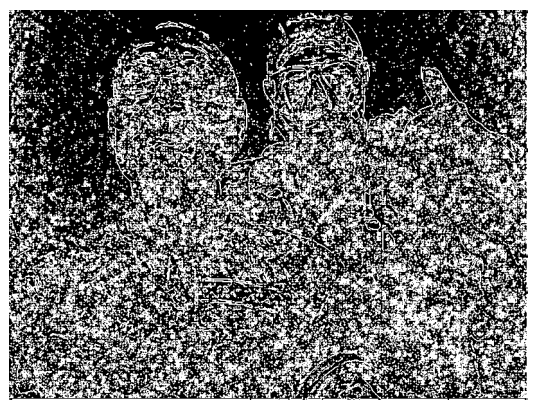

(a)

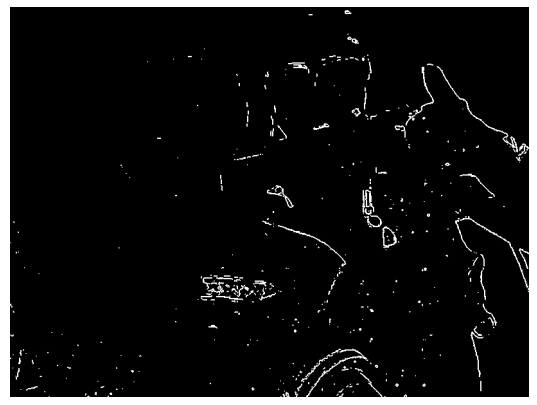

(d)

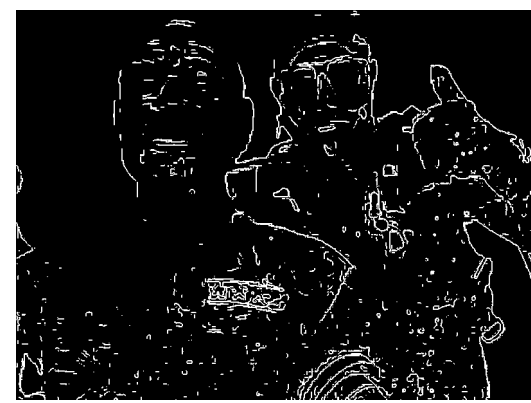

(b)

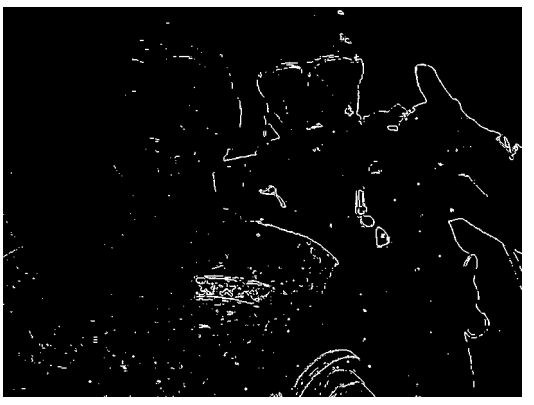

(e)

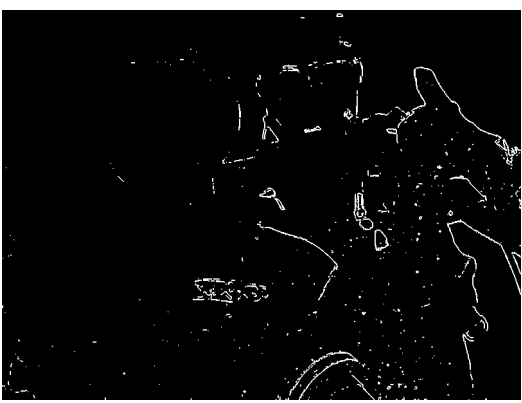

(c)

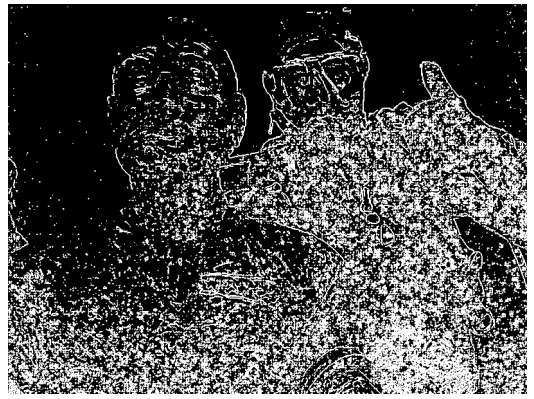

(f)

Figure 7: Corresponding visible edge maps of the recovered Diver image. (a) (9) (b) (25) (c) (18) (d) (19) (e) (26) (f) Red Channel algorithm.

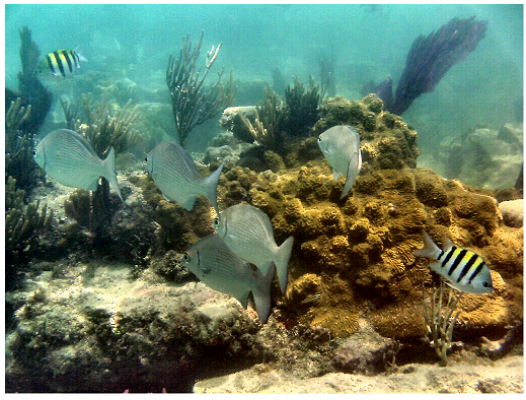

(a)

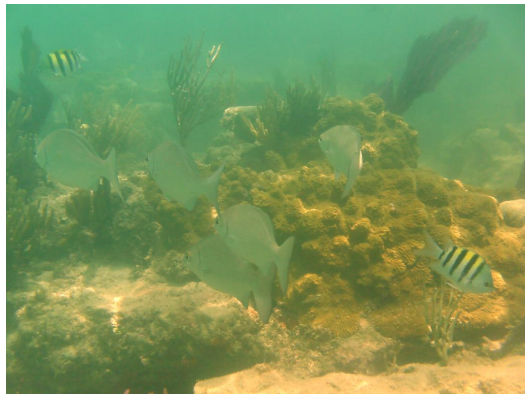

(d)

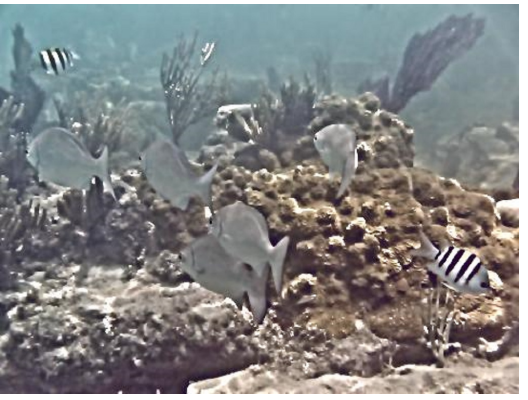

(b)

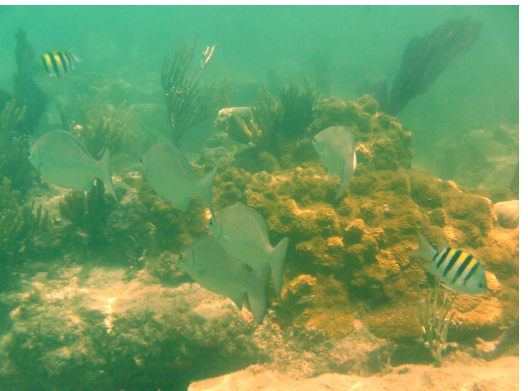

(e)

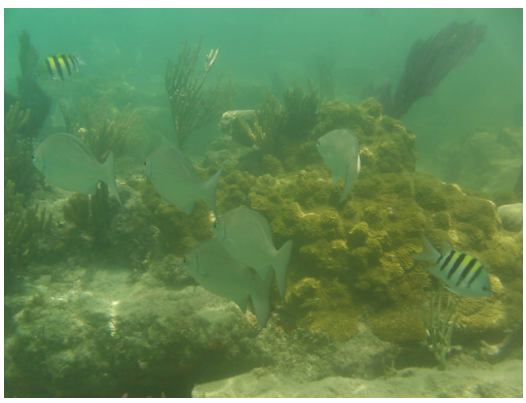

(c)

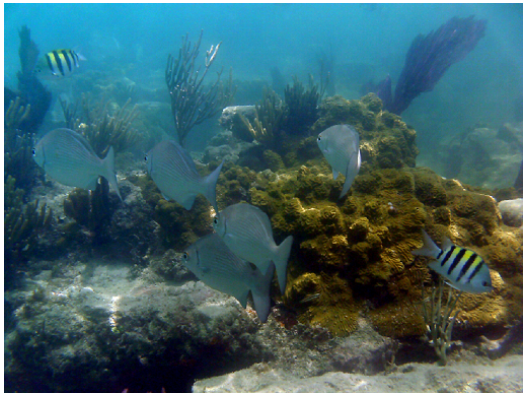

(f)

Figure 8: Results on the Fishes image. (a) With (9) (b) With (25) (c) With (18) (d) With (19) (e) With (26) (f) With the Red Channel algorithm. 


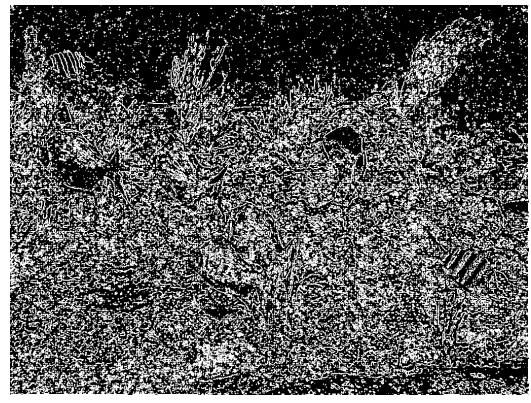

(a)

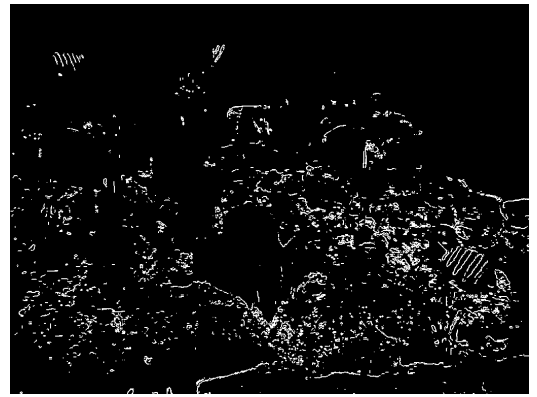

(d)

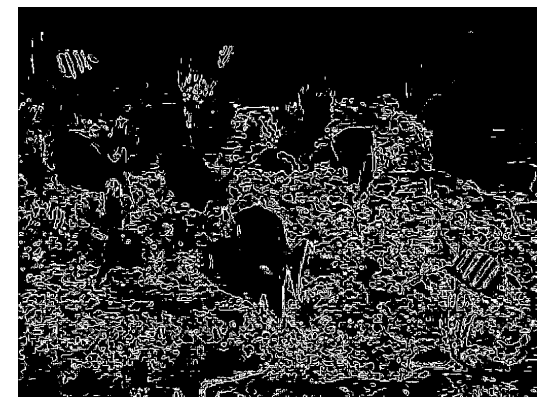

(b)

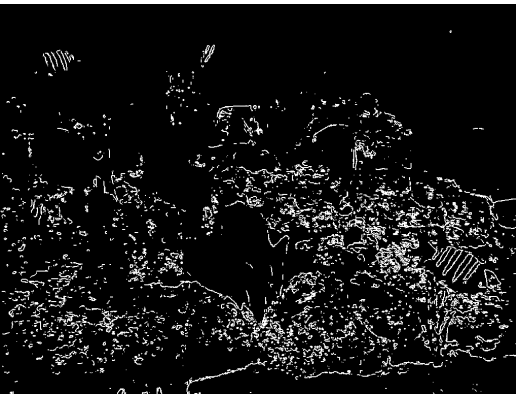

(e)

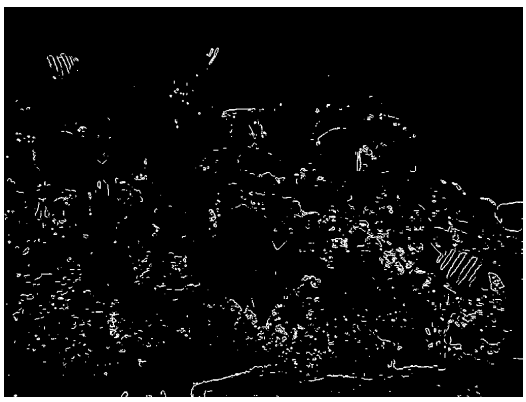

(c)

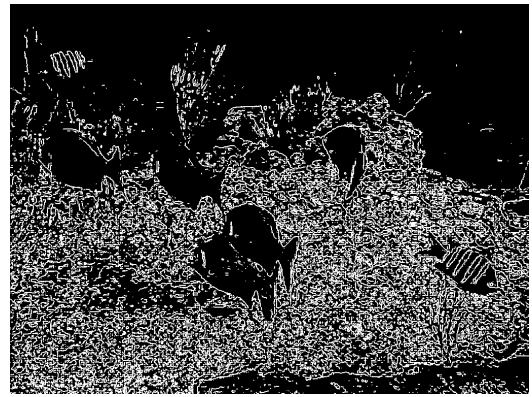

(f)

Figure 9: Corresponding visible edge maps of the recovered Fishes image. (a) (9) (b) (25) (c) (18) (d) (19) (e) (26) (f) Red Channel algorithm.

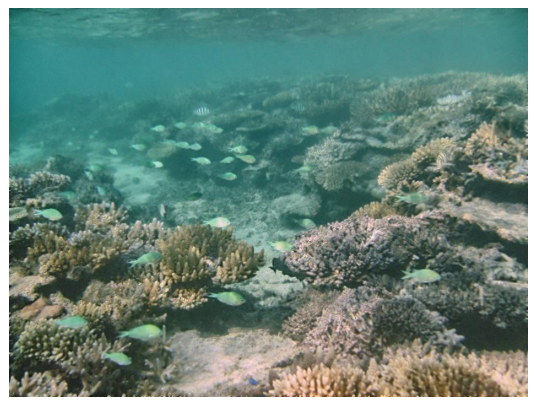

(a)

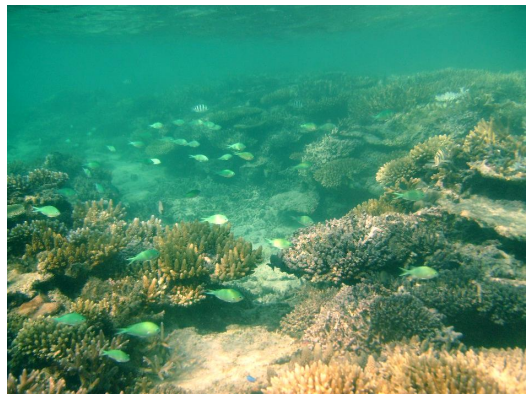

(d)

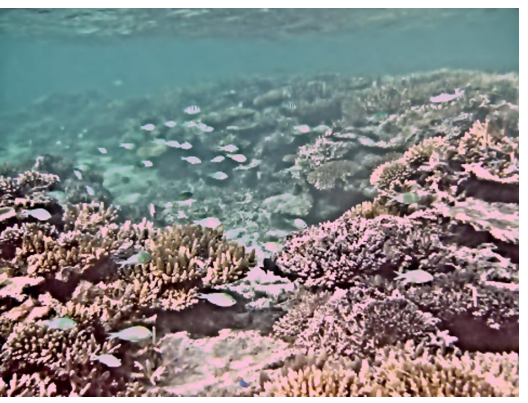

(b)

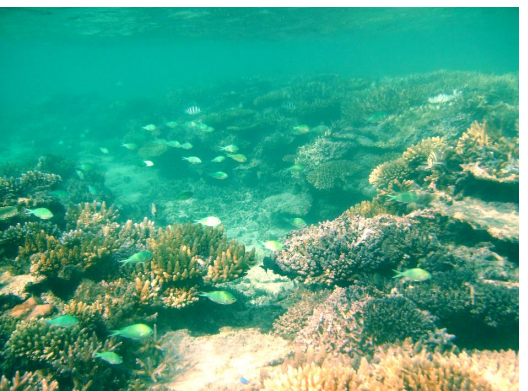

(e)

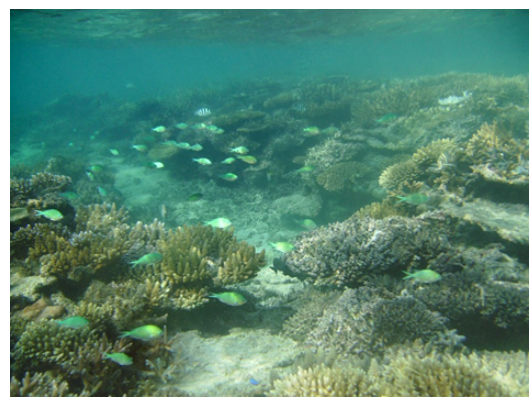

(c)

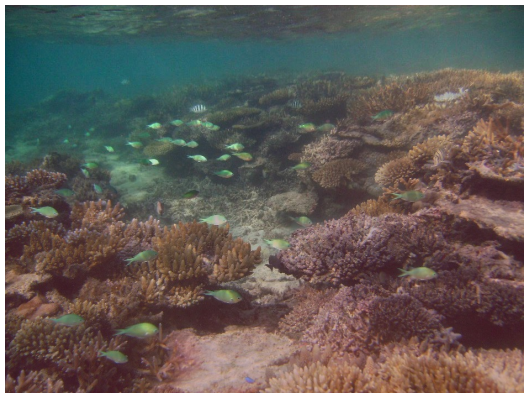

(f)

Figure 10: Results on the Open scene image. (a) With (9) (b) With (25) (c) With (18) (d) With (19) (e) With (26) (f) With the Red Channel algorithm. 


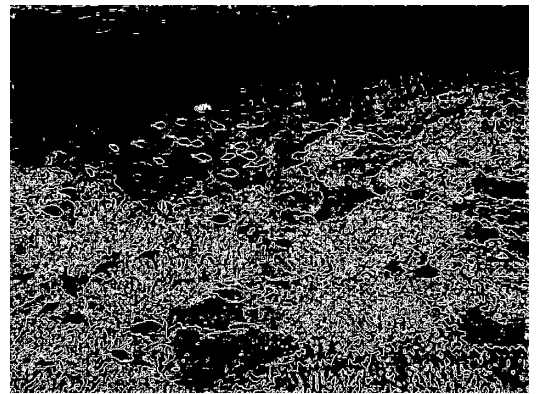

(a)

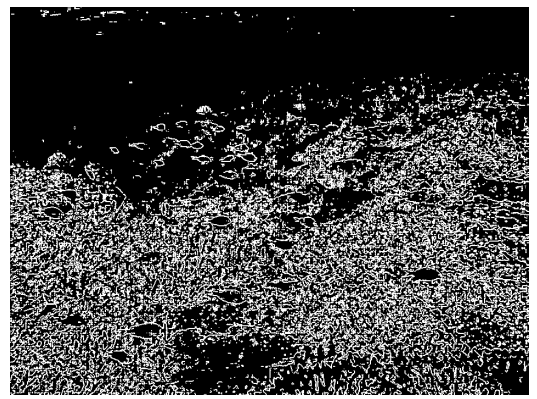

(d)

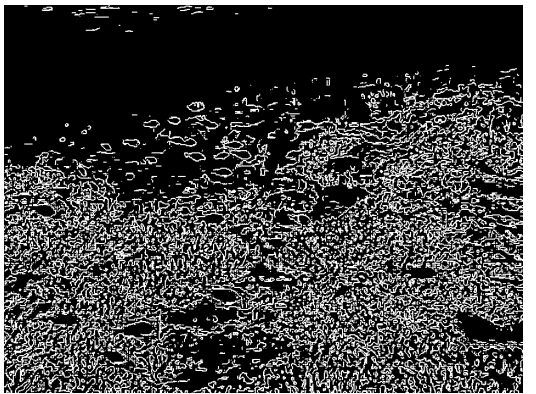

(b)

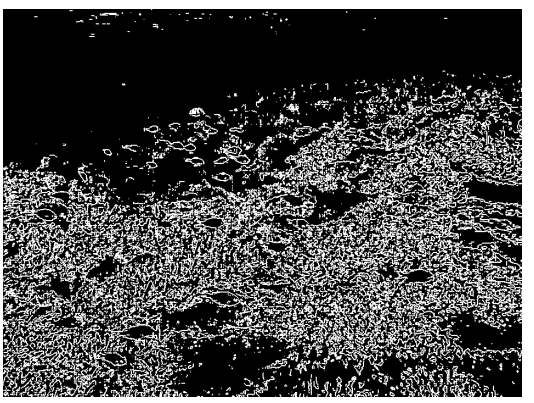

(e)

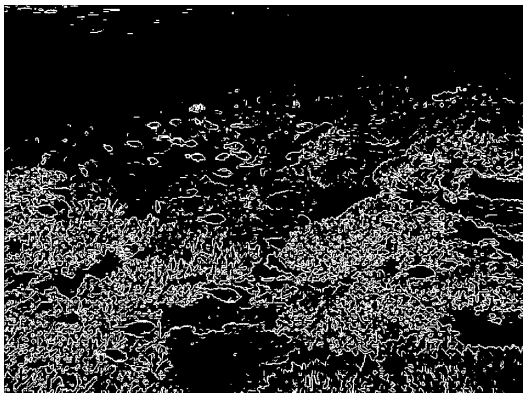

(c)

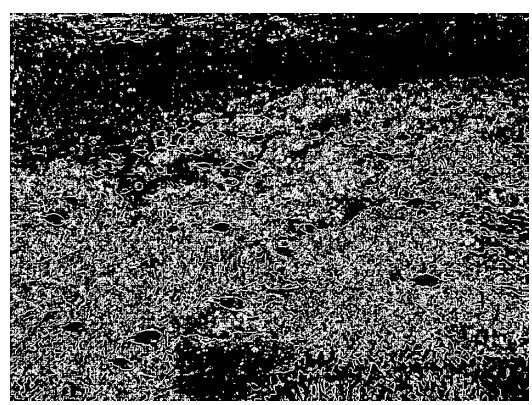

(f)

Figure 11: Corresponding visible edge maps of the Open scene image. (a) (9) (b) (25) (c) (18) (d) (19) (e) (26) (f) Red Channel algorithm.

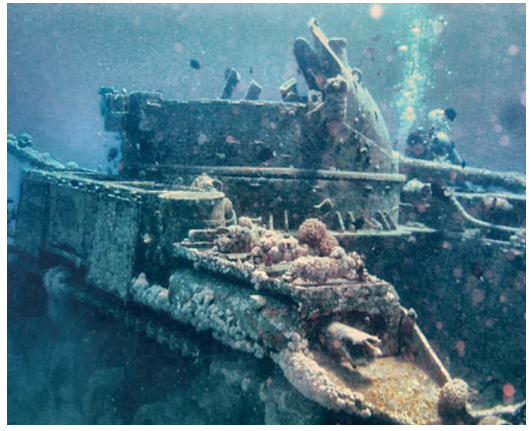

(a)

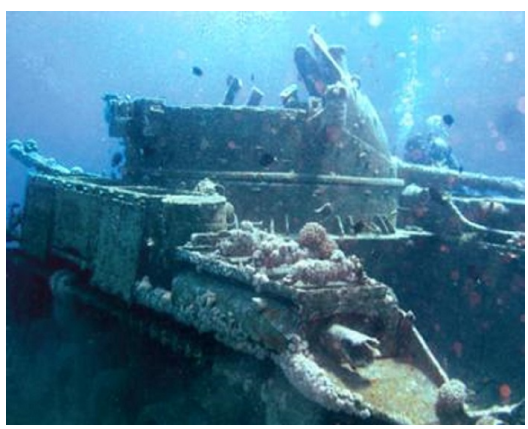

(d)

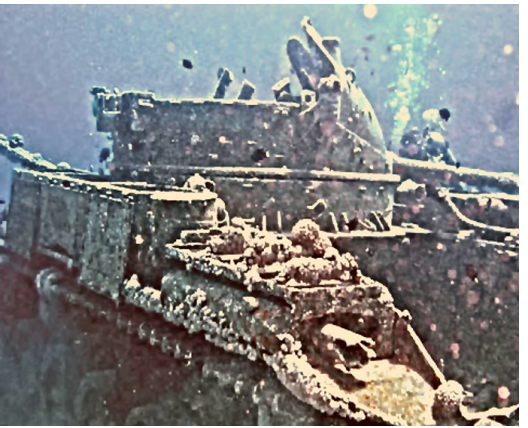

(b)

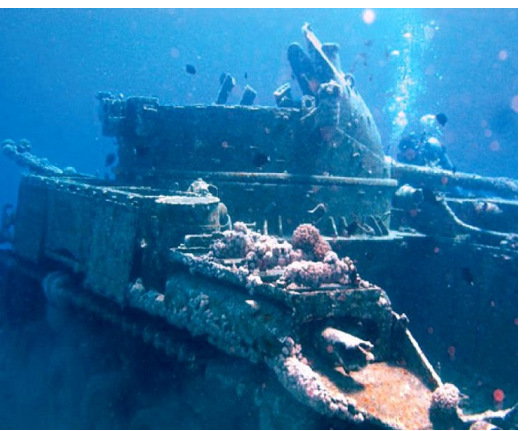

(e)

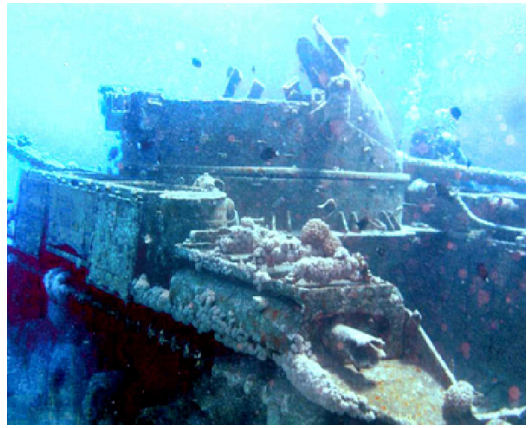

(c)

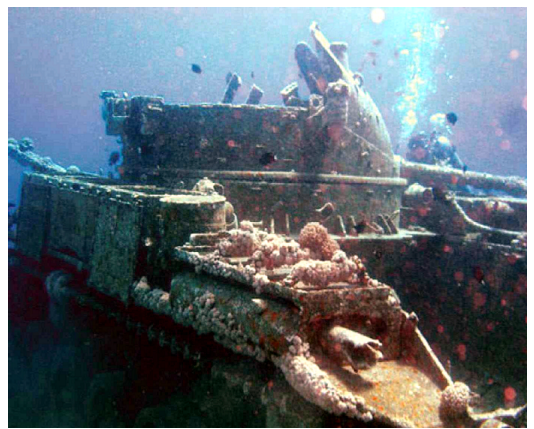

(f)

Figure 12: Results on the Ship image. (a) With (9) (b) With (25) (c) With (18) (d) With (19) (e) With (26) (f) With the Red Channel algorithm. 


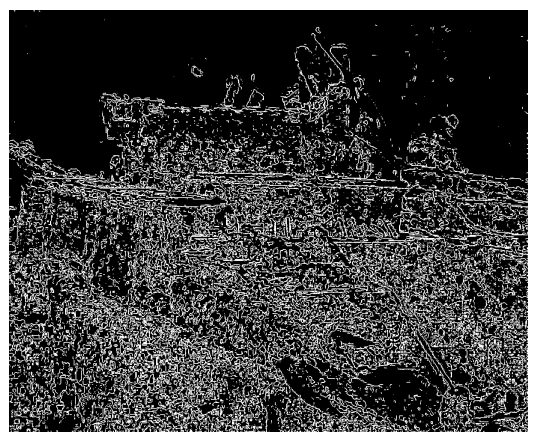

(a)

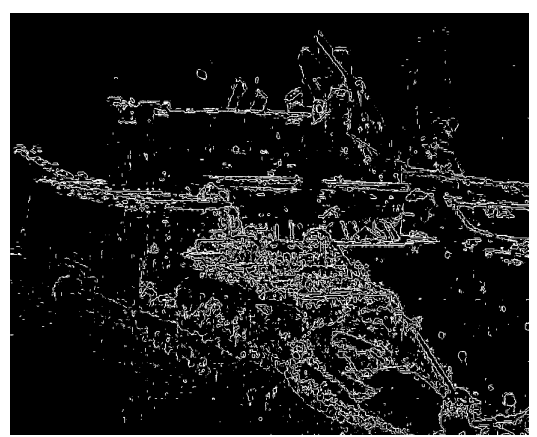

(d)

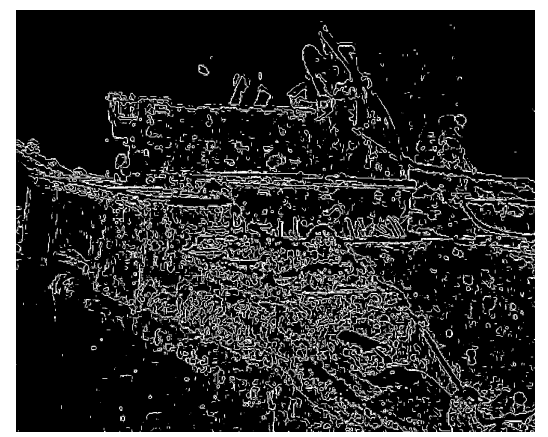

(b)

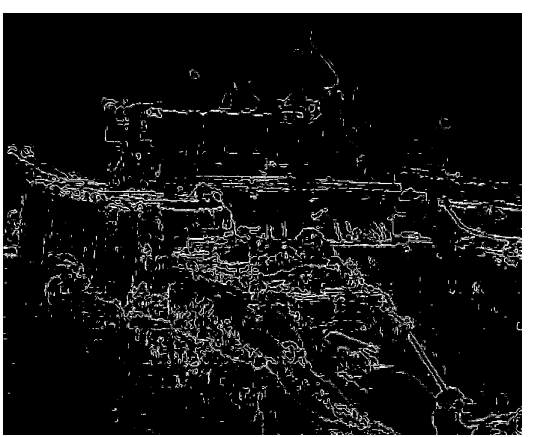

(e)

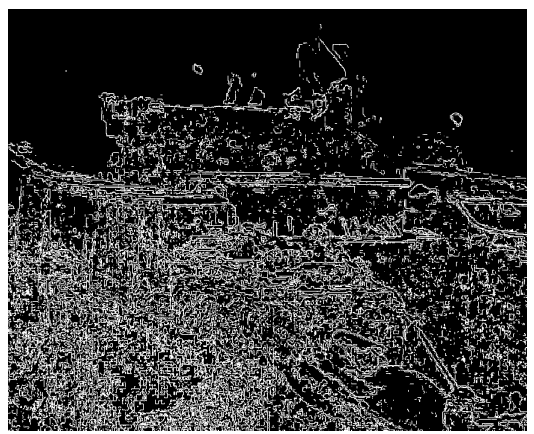

(c)

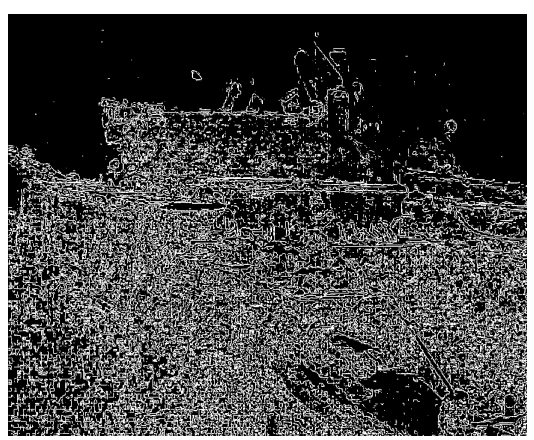

(f)

Figure 13: Corresponding visible edge maps of the Ship image. (a) (9) (b) (25) (c) (18) (d) (19) (e) (26) (f) Red Channel algorithm.

dispersion of the three $R G B$ channels, $\mu_{R}, \mu_{G}, \mu_{B}$, and of its standard deviations, $\sigma_{R}, \sigma_{G}, \sigma_{B}$. To quantify this dispersion, we simply pick the maximum of their mutual distances, i.e.:

$$
\mu_{\mathrm{diff}}=\max \left(\left|\mu_{R}-\mu_{G}\right|,\left|\mu_{R}-\mu_{B}\right|,\left|\mu_{B}-\mu_{G}\right|\right)
$$

and

$$
\sigma_{\mathrm{diff}}=\max \left(\left|\sigma_{R}-\sigma_{G}\right|,\left|\sigma_{R}-\sigma_{B}\right|,\left|\sigma_{B}-\sigma_{G}\right|\right)
$$

By enforcing that these coefficients remain relatively low, we are requiring that color cast and dominance are reduced. On the other hand, notice that a completely gray image would achieve the lowest score in both $\mu_{\text {diff }}$ and $\sigma_{\text {diff }}$. To incorporate the requirement of recovering a variety of colors, we can simply consider a measure of saturation. To keep the consistency of requiring low value to reflect optimal behavior, we introduce a third coefficient, given by:

$$
\lambda=1-\operatorname{mean}(\operatorname{Sat}(\overrightarrow{\mathrm{I}})),
$$

where $\operatorname{Sat}(\overrightarrow{\mathrm{I}})$ is defined as in Eq. 11 . Each of these metrics measures the ability to reduce color dominance, remove color cast and retrieve rich colors, respectively.

Regarding color dominance, Fig. (14) reproduces the scores that each method achieved in the metric given by Eq. (13), for each image in our test set. We see that the method in (25) is able to carry the chromatic means to a common value, obtaining the best score in color dominance reduction. Ancuti's algorithm (9) and ours are ranked in second/third position here, while the rest of the methods fail to remove color dominance.

Regarding color cast reduction, again the method in (25) scores in the best positions, only beaten by our method on Image 1 and obtaining similar results as (26) on Image 3.

However, these results should be judged cautiously. The algorithm in (25) removes color dominance and cast by sacrificing the recovery of intense colors, i.e., it is obtaining rather grayish images, see for example Fig. 8b. This is 

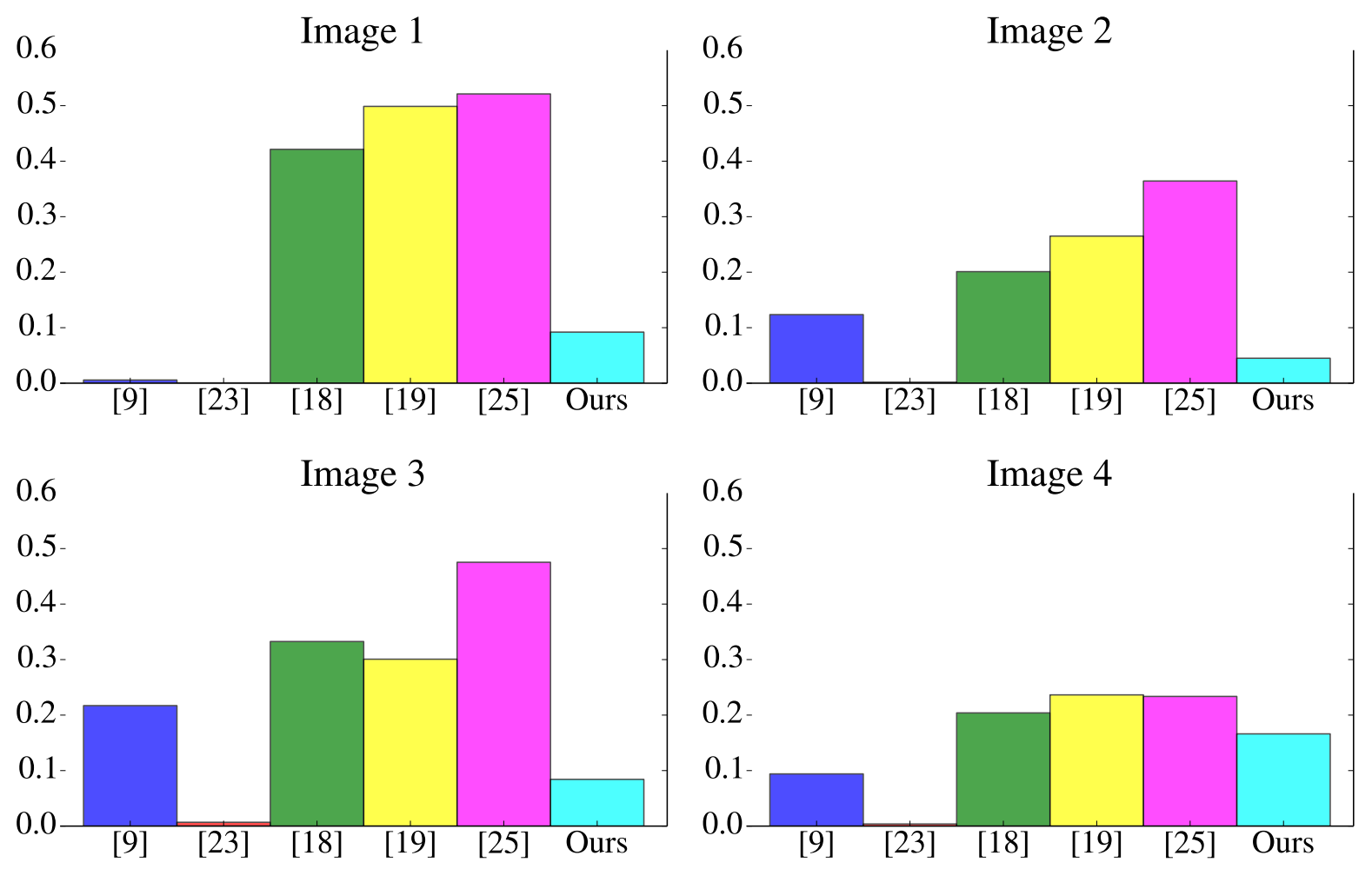

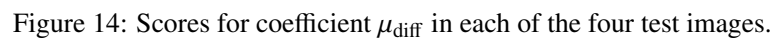

confirmed by inspection of Fig. 16. Therein, we realize that (25) is the method that recovers the least vivid colors. On the other hand, the methods in (18), (19) and (26) are obtaining very saturated colors, but clearly sacrificing the reduction of color dominance, and to some extent, also color cast. We thus see that, among the three methods that are able to reduce color dominance and color cast, Ancuti's method (9) and ours are again ranked as the first/second with respect to the ability to retrieve intense colors. This quantitative evaluation seems to soundly match perceptual criteria, as informal inspection of the experimental results in the previous section shows.

\section{Discussion and Conclusion}

In this work, we have proposed a new method for the restoration of underwater images that tackles both visibility loss and color corruption. Our work extends the Dark Channel Method, adapting to the way these images are degraded. We provide also a general methodology to locate artificially illuminated areas within an underwater scene, and suggest a method to handle these regions properly, avoiding color artifacts that can appear due to the wrong estimate of depth in them.

Regarding the experimental results, we have proposed to perform a twofold evaluation. First, to measure the improvement of image contrast, we resort to a metric proposed in (23) to quantify visibility enhancement on images degraded by fog. Second, to evaluate the quality of the recovered colors, we propose to use three basic indicators that adapt nicely to the problematic of assessing the quality of underwater colors after image restoration.

Images restored with our method have been compared with five different state-of-the-art algorithms. Results show that our approach obtains good-quality images, with a visibility enhancement comparable or better than other recent methods. As for color recovery, we retrieve natural colors, consistently ranking among the best positions among different images, regardless of the different water conditions. 


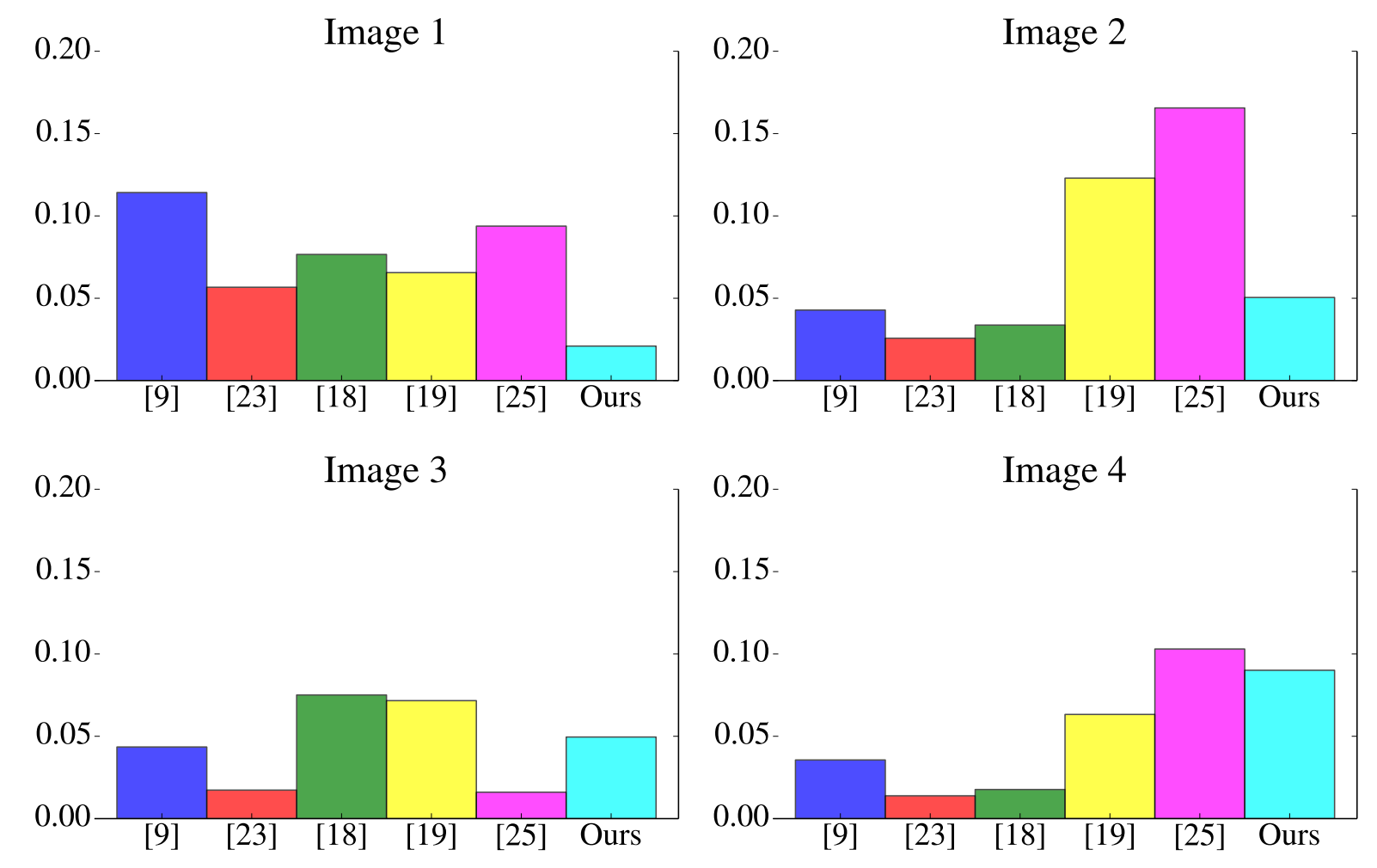

Figure 15: Scores for coefficient $\sigma_{\text {diff }}$ in each of the four test images. 

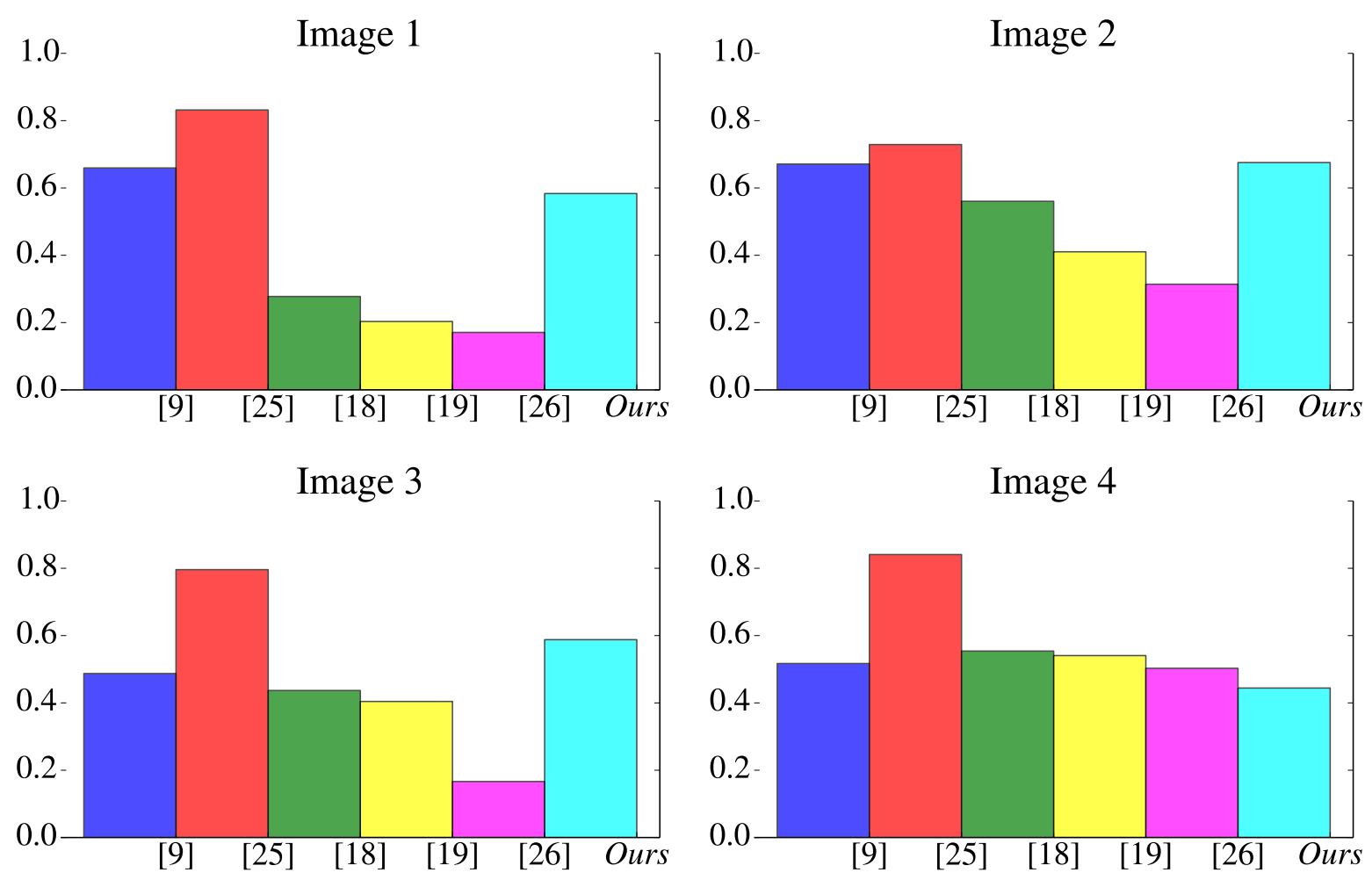

Figure 16: Scores for coefficient $\lambda$ in each of the four test images.

We have carried out our experiments on four different test images that attempt to capture the wide variability of underwater images. Although our test set encompasses rather different images, we are aware that it is impossible to reflect the whole range of possibilities regarding the different water qualities, depth from the atmosphere, illuminations and a large number of parameters that affect underwater image acquisition. To improve potential comparison and evaluation, we have set up an online repository ${ }^{1}$ where the reader can find all the images that appear in this paper at full resolution, as well as our restoration results in a wider set of test images, and code to generate Figures (14), (15) and $(16)$, for comparison purposes.

\section{Appendix}

\section{Proof of Theorem 2.2}

Departing from Eqs. (3), we divide by the waterlight:

$$
\left(\frac{\mathbf{1}-\mathrm{I}^{R}}{\mathbf{1}-\mathrm{A}^{R}}, \frac{\mathrm{I}^{G}}{\mathrm{~A}^{G}}, \frac{\mathrm{I}^{B}}{\mathrm{~A}^{B}}\right)=\left(\mathrm{t} \frac{\mathbf{1}-\mathrm{J}^{R}}{\mathbf{1}-\mathrm{A}^{R}}+\mathbf{1}-\mathrm{t}, \mathrm{t} \frac{\mathrm{J}^{G}}{\mathrm{~A}^{G}}+\mathbf{1}-\mathrm{t}, \mathrm{t} \frac{\mathrm{J}^{B}}{\mathrm{~A}^{B}}+\mathbf{1}-\mathrm{t}\right),
$$

and we take minima over local neighborhoods on every image. Recall waterlight are scalar images build out of constant values, so they can be extracted from the minima operators. This is also true for the estimate of the transmission

\footnotetext{
${ }^{1}$ https://github.com/agaldran/UnderWater
} 
map, that we consider to be a locally constant image, $\min _{\Omega(x)} \widetilde{\mathrm{t}}(x)=\widetilde{\mathrm{t}}(x)$, so we obtain:

$$
\begin{aligned}
& \left(\frac{\min _{\Omega}\left(\mathbf{1}-\mathrm{I}^{R}\right)}{\mathbf{1}-\mathrm{A}^{R}}, \frac{\min _{\Omega} \mathrm{I}^{G}}{\mathrm{~A}^{G}}, \frac{\min _{\Omega} \mathrm{I}^{B}}{\mathrm{~A}^{B}}\right)= \\
& \left(\widetilde{\mathrm{t}} \frac{\min \left(\left(\mathbf{1}-\mathrm{J}^{R}\right)\right)}{\mathbf{1}-\mathrm{A}^{R}}+\mathbf{1}-\widetilde{\mathrm{t}}, \widetilde{\mathrm{t}} \frac{\min _{\Omega} \mathrm{J}^{G}}{\mathrm{~A}^{G}}+\mathbf{1}-\widetilde{\mathrm{t}}, \widetilde{\mathrm{t}} \frac{\min _{\Omega}}{\mathrm{A}^{B}}+\mathbf{1}-\widetilde{\mathrm{t}}\right)
\end{aligned}
$$

After taking minima at both sides of the above equation, we deduce:

$$
\begin{aligned}
& \min \left(\frac{\min _{\Omega}\left(\mathbf{1}-\mathrm{I}^{R}\right)}{\mathbf{1}-\mathrm{A}^{R}}, \frac{\min _{\Omega} \mathrm{I}^{G}}{\mathrm{~A}^{G}}, \frac{\min _{\Omega} \mathrm{I}^{B}}{\mathrm{~A}^{B}}\right)= \\
& \widetilde{\mathrm{t}} \min \left(\frac{\min _{\Omega}\left(\left(\mathbf{1}-\mathrm{J}^{R}\right)\right)}{\mathbf{1}-\mathrm{A}^{R}}, \frac{\min _{\Omega}\left(\mathrm{J}^{G}\right)}{\mathrm{A}^{G}}, \frac{\min _{\Omega}\left(\mathrm{J}^{B}\right)}{\mathrm{A}^{B}}\right)+\mathbf{1}-\widetilde{\mathrm{t}}
\end{aligned}
$$

Direct application now of the Red Channel prior (4) cancels out the first term of the right hand side of the above equation. Simple rearrangement leads to the desired conclusion.

\section{Proof of Theorem 2.3}

According to Eq. (5), it is enough to prove that:

$$
0 \leq \min \left(\frac{\min _{\Omega(x)} \mathbf{1}-\mathrm{I}^{R}(x)}{\mathbf{1}-\mathrm{A}^{R}}, \frac{\min _{\Omega(x)} \mathrm{I}^{G}(x)}{\mathrm{A}^{G}}, \frac{\min _{\Omega(x)} \mathrm{I}^{B}(x)}{\mathrm{A}^{B}}\right) \leq 1,
$$

for every pixel $x$. Being every quantity in Ineq. A1 positive, the first inequality is automatically verified. To see that the inner expression is bounded by 1 , let us recall that Airlight $\overrightarrow{\mathrm{A}}$ was defined to be located at the pixel $x_{0}$ verifying that:

$$
\overrightarrow{\mathrm{A}}=\left(\mathrm{A}^{R}, \mathrm{~A}^{G}, \mathrm{~A}^{B}\right)=\left(\mathrm{I}^{R}\left(x_{0}\right), \mathrm{I}^{G}\left(x_{0}\right), \mathrm{I}^{B}\left(x_{0}\right)\right) \text { s.t. } \mathrm{I}^{R E D}\left(x_{0}\right) \geq \mathrm{I}^{R E D}(x) \quad \forall x .
$$

Then, we take an arbitrary pixel location $x^{*}$, and we apply the definition of the Red Channel given in Eq.4 to the degraded image $\overrightarrow{\mathrm{I}}$ :

$$
\begin{aligned}
& \min \left(\min _{y \in \Omega\left(x^{*}\right)}\left(\mathbf{1}-\mathrm{I}^{R}(y)\right), \min _{y \in \Omega\left(x^{*}\right)}\left(\mathrm{I}^{G}(y)\right), \min _{y \in \Omega\left(x^{*}\right)}\left(\mathrm{I}^{B}(y)\right)\right) \leq \\
& \min \left(\min _{y \in \Omega\left(x_{0}\right)}\left(\mathbf{1}-\mathrm{I}^{R}(y)\right), \min _{y \in \Omega\left(x_{0}\right)}\left(\mathrm{I}^{G}(y)\right), \min _{y \in \Omega\left(x_{0}\right)}\left(\mathrm{I}^{B}(y)\right)\right)
\end{aligned}
$$

Let us now notice the fact that, since we always have $x_{0} \in \Omega\left(x_{0}\right)$, we can write:

$$
\begin{aligned}
& \min _{y \in \Omega\left(x_{0}\right)}\left(\mathbf{1}-\mathrm{I}^{R}(y)\right) \leq \mathbf{1}-\mathrm{I}^{R}\left(x_{0}\right), \\
& \min _{y \in \Omega\left(x_{0}\right)} \mathrm{I}^{G}(y) \leq \mathrm{I}^{G}\left(x_{0}\right), \\
& \min _{y \in \Omega\left(x_{0}\right)} \mathrm{I}^{B}(y) \leq \mathrm{I}^{B}\left(x_{0}\right) .
\end{aligned}
$$


With this, we can rewrite Ineq. $\mathrm{A} 2]$ as:

$$
\begin{gathered}
\min \left(\min _{y \in \Omega\left(x^{*}\right)}\left(\mathbf{1}-\mathrm{I}^{R}(y)\right), \min _{y \in \Omega\left(x^{*}\right)} \mathrm{I}^{G}(y), \min _{y \in \Omega\left(x^{*}\right)}\left(\mathrm{I}^{B}(y)\right)\right) \leq \\
\min \left(\mathbf{1}-\mathrm{I}^{R}\left(x_{0}\right), \mathrm{I}^{G}\left(x_{0}\right), \mathrm{I}^{B}\left(x_{0}\right)\right)=\min \left(1-\mathrm{A}^{R}, \mathrm{~A}^{G}, \mathrm{~A}^{B}\right) .
\end{gathered}
$$

Now, for the pixel $x^{*}$, there are three possibilities regarding the left-hand-side of Ineq. [A3], depending on which of the three inner quantities attains the minimum.

First Possibility: Suppose it is the first quantity, i.e.:

$$
\min \left(\min _{y \in \Omega\left(x^{*}\right)}\left(\mathbf{1}-\mathrm{I}^{R}(y)\right), \min _{y \in \Omega\left(x^{*}\right)}\left(\mathrm{I}^{G}(y)\right), \min _{y \in \Omega\left(x^{*}\right)}\left(\mathrm{I}^{B}(y)\right)\right)=\min _{y \in \Omega\left(x^{*}\right)}\left(\mathbf{1}-\mathrm{I}^{R}(y)\right)
$$

In this case, Eq. A3 can be used to write:

$$
\min _{y \in \Omega\left(x^{*}\right)}\left(1-\mathrm{I}^{R}(y)\right) \leq \min \left(1-\mathrm{A}^{R}, \mathrm{~A}^{G}, \mathrm{~A}^{B}\right) \leq 1-\mathrm{A}^{R}
$$

and we deduce that:

$$
\frac{\min _{y \in \Omega\left(x^{*}\right)}\left(1-\mathrm{I}^{R}(y)\right)}{\mathbf{1}-\mathrm{A}^{R}} \leq 1
$$

Finally, using Ineq. A4, we arrive to the desired conclusion:

$$
\min \left(\frac{\min _{y \in \Omega\left(x^{*}\right)} \mathbf{1}-\mathrm{I}^{R}(y)}{\mathbf{1}-\mathrm{A}^{R}}, \frac{\min _{y \in \Omega\left(x^{*}\right)} \mathrm{I}^{G}(y)}{\mathrm{A}^{G}}, \frac{\min _{y \in \Omega\left(x^{*}\right)} \mathrm{I}^{B}(y)}{\mathrm{A}^{B}}\right) \leq \frac{\min _{y \in \Omega\left(x^{*}\right)}\left(\mathbf{1}-\mathrm{I}^{R}(y)\right)}{\mathbf{1}-\mathrm{A}^{R}} \leq 1 .
$$

Second Possibility:. Suppose now the minimum on the left hand side of Ineq. $\mathrm{A} 3$ is attained by $\min _{y \in \Omega(x)} \mathrm{I}^{G}(y)$. Just as in the first case, we can write:

$$
\min _{y \in \Omega\left(x^{*}\right)} \mathrm{I}^{G}(y) \leq \min \left(1-\mathrm{A}^{R}, \mathrm{~A}^{G}, \mathrm{~A}^{B}\right) \leq \mathrm{A}^{G},
$$

and we deduce now that:

$$
\frac{\min _{y \in \Omega\left(x^{*}\right)} \mathrm{I}^{G}(y)}{\mathrm{A}^{G}} \leq 1,
$$

which leads us to:

$$
\min \left(\frac{\min _{y \in \Omega\left(x^{*}\right)} 1-\mathrm{I}^{R}(y)}{\mathbf{1}-\mathrm{A}^{R}}, \frac{\min _{y \in \Omega\left(x^{*}\right)} \mathrm{I}^{G}(y)}{\mathrm{A}^{G}}, \frac{\min _{y \in \Omega\left(x^{*}\right)} \mathrm{I}^{B}(y)}{\mathrm{A}^{B}}\right) \leq \frac{\min _{y \in \Omega\left(x^{*}\right)} \mathrm{I}^{G}(y)}{\mathrm{A}^{G}} \leq 1 .
$$

Third Possibility: If the minimum in the left hand side of Ineq. $\mathrm{A} 3$ is attained by $\min _{y \in \Omega\left(x^{*}\right)} \mathrm{I}^{B}(y)$, similar derivations as in the previous cases lead us to:

$$
\min \left(\frac{\min _{y \in \Omega\left(x^{*}\right)} \mathbf{1}-\mathrm{I}^{R}(y)}{\mathbf{1}-\mathrm{A}^{R}}, \frac{\min _{y \in \Omega\left(x^{*}\right)} \mathrm{I}^{G}(y)}{\mathrm{A}^{G}}, \frac{\min _{y \in \Omega\left(x^{*}\right)} \mathrm{I}^{B}(y)}{\mathrm{A}^{B}}\right) \leq \frac{\min _{y \in \Omega\left(x^{*}\right)} \mathrm{I}^{B}(y)}{\mathrm{A}^{B}} \leq 1 .
$$

With any of the three possibilities, the hypothesis of the theorem is verified. As the choice of pixel $x^{*}$ was arbitrary, this concludes the proof. 


\section{Proof of Theorem 2.4}

Departing from Eqs. (6), we divide by the waterlight:

$$
\left(\frac{\mathbf{1}-\mathrm{I}^{R}}{\mathbf{1}-\mathrm{A}^{R}}, \frac{\mathrm{I}^{G}}{\mathrm{~A}^{G}}, \frac{\mathrm{I}^{B}}{\mathrm{~A}^{B}}\right)=\left(\mathrm{t}^{R} \frac{\mathbf{1}-\mathrm{J}^{R}}{\mathbf{1}-\mathrm{A}^{R}}+\mathbf{1}-\mathrm{t}^{R}, \mathrm{t}^{G} \frac{\mathrm{J}^{G}}{\mathrm{~A}^{G}}+\mathbf{1}-\mathrm{t}^{G}, \mathrm{t}^{B} \frac{\mathrm{J}^{B}}{\mathrm{~A}^{B}}+\mathbf{1}-\mathrm{t}^{B}\right),
$$

and we take minima over local neighborhoods on every image. As in the proof of Theorem [2.2], waterlight and transmission map estimates can be extracted from the minima operators, since they are locally constant images, yielding:

$$
\begin{aligned}
& \left(\frac{\min _{\Omega}\left(\mathbf{1}-\mathrm{I}^{R}\right)}{\mathbf{1}-\mathrm{A}^{R}}, \frac{\min _{\Omega} \mathrm{I}^{G}}{\mathrm{~A}^{G}}, \frac{\min _{\Omega} \mathrm{I}^{B}}{\mathrm{~A}^{B}}\right)= \\
& \left(\mathrm{t}^{R} \frac{\min \left(\left(\mathbf{1}-\mathrm{J}^{R}\right)\right)}{\mathbf{1}-\mathrm{A}^{R}}+\mathbf{1}-\mathrm{t}^{R}, \mathrm{t}^{G} \frac{\min _{\Omega}\left(\mathrm{J}^{G}\right)}{\mathrm{A}^{G}}+\mathbf{1}-\mathrm{t}^{G}, \mathrm{t}^{B} \frac{\min \left(\mathrm{J}^{B}\right)}{\mathrm{A}^{B}}+\mathbf{1}-\mathrm{t}^{B}\right)
\end{aligned}
$$

We want to compute the minimum of the three components of both sides of (A5). Notice that in our case, the transmission maps can only be extracted from this second minimum operator after the following manipulations:

$$
\begin{aligned}
\mathrm{t}^{G} \frac{\min _{\Omega} \mathrm{J}^{G}}{\mathrm{~A}^{G}}+\mathbf{1}-\mathrm{t}^{G} & =\mathrm{t}_{R} \mathrm{t}_{R}^{\alpha_{G}-1} \frac{\min _{\Omega} \mathrm{J}^{G}}{\mathrm{~A}^{G}}+\mathbf{1}-\mathrm{t}_{R}+\left(\mathrm{t}_{R}-\mathrm{t}_{R}^{\alpha_{G}}\right) \\
& =\mathrm{t}_{R} \mathrm{t}_{R}^{\alpha_{G}-1} \frac{\min _{\Omega} \mathrm{J}^{G}}{\mathrm{~A}^{G}}+\mathbf{1}-\mathrm{t}_{R}+\mathrm{t}_{R}\left(\mathbf{1}-\mathrm{t}_{R}^{\alpha_{G}-1}\right) \\
& =\mathrm{t}_{R}\left(\mathrm{t}_{R}^{\alpha_{G}-1} \frac{\min _{\Omega} \mathrm{J}^{G}}{\mathrm{~A}^{G}}+\left(\mathbf{1}-\mathrm{t}_{R}^{\alpha_{G}-1}\right)\right)+\mathbf{1}-\mathrm{t}_{R}
\end{aligned}
$$

and analogously,

$$
\mathrm{t}^{B} \frac{\min _{\Omega} \mathrm{J}^{B}}{\mathrm{~A}^{B}}+\mathbf{1}-\mathrm{t}^{B}=\mathrm{t}_{R}\left(\mathfrak{t}_{R}^{\alpha_{B}-1} \frac{\min _{\Omega} \mathbf{J}^{B}}{\mathrm{~A}^{B}}\left(\mathbf{1}-\mathrm{t}_{R}^{\alpha_{B}-1}\right)\right)+\mathbf{1}-\mathrm{t}_{R}
$$

where (7) has been used.

Inserting that in the above equation, Eq. A5] becomes:

$$
\begin{aligned}
& \left(\frac{\min _{\Omega}\left(\mathbf{1}-\mathrm{I}^{R}\right)}{\mathbf{1}-\mathrm{A}^{R}}, \frac{\min _{\Omega} \mathrm{I}^{G}}{\mathrm{~A}^{G}}, \frac{\min _{\Omega} \mathrm{I}^{B}}{\mathrm{~A}^{B}}\right)= \\
& \mathrm{t}^{R}\left(\frac{\min _{\Omega}\left(\left(\mathbf{1}-\mathrm{J}^{R}\right)\right)}{\mathbf{1}-\mathrm{A}^{R}}, \mathrm{t}_{G}^{\lambda_{R}-1} \frac{\min _{\Omega}\left(\mathrm{J}^{G}\right)}{\mathrm{A}^{G}}+\mathbf{1}-\mathrm{t}_{R}^{\lambda_{R}-1},\right. \\
& \left.\mathrm{t}^{\lambda_{B}-1} \frac{\min _{\Omega}\left(\mathrm{J}^{B}\right)}{\mathrm{A}^{B}}+\mathbf{1}-\mathrm{t}^{\lambda_{R}-1}\right)+(\mathbf{1}, \mathbf{1}, \mathbf{1})-\left(\mathrm{t}_{R}, \mathrm{t}_{R}, \mathrm{t}_{R}\right) .
\end{aligned}
$$


Now we can take the minimum on both sides of Eq. A6:

$$
\begin{aligned}
& \min \left(\frac{\min _{\Omega}\left(\mathbf{1}-\mathrm{I}^{R}\right)}{\mathbf{1}-\mathrm{A}^{R}}, \frac{\min _{\Omega} \mathrm{I}^{G}}{\mathrm{~A}^{G}}, \frac{\min _{\Omega} \mathrm{I}^{B}}{\mathrm{~A}^{B}}\right)= \\
& \mathrm{t}^{R} \min \left(\frac{\min _{\Omega}\left(\left(\mathbf{1}-\mathrm{J}^{R}\right)\right)}{\mathbf{1}-\mathrm{A}^{R}}, \mathrm{t}_{G}^{\lambda_{R}-1} \frac{\min _{\Omega}\left(\mathrm{J}^{G}\right)}{\mathrm{A}^{G}}+\right. \\
& \left.\mathbf{1}-\mathrm{t}_{R}^{\lambda_{R}-1}, \mathrm{t}^{\lambda_{B}-1} \frac{\min _{\Omega}\left(\mathrm{J}^{B}\right)}{\mathrm{A}^{B}}+\mathbf{1}-\mathrm{t}^{\lambda_{R}-1}\right)+\mathbf{1}-\mathrm{t}_{R} .
\end{aligned}
$$

Here the vector multiplying $t_{R}$ cancels out due to the hypothesis of the generalized red channel prior 88 , and we obtain:

$$
\mathrm{t}^{R}(x)=\mathbf{1}-\min \left(\frac{\min _{\Omega} \mathbf{1}-\mathrm{I}^{R}}{\mathbf{1}-\mathrm{A}^{R}}, \frac{\min _{\Omega} \mathrm{I}^{G}}{\mathrm{~A}^{G}}, \frac{\min _{\Omega} \mathrm{I}^{B}}{\mathrm{~A}^{B}}\right) .
$$

And the other two transmission maps are immediately deduced from Eqs. (7).

\section{Acknowledgment}

David Pardo was partially funded by the Project of the Spanish Ministry of Economy and Competitiveness with reference MTM2013-40824-P, the BCAM "Severo Ochoa" accreditation of excellence SEV-2013-0323, the CYTED 2011 project 712RT0449, and the Basque Government Consolidated Research Group Grant IT649-13 on "Mathematical Modeling, Simulation, and Industrial Applications (M2SI)".

The authors would also like to thank Nicholas Carlevaris-Bianco and Huimin Lu for kindly providing with the code of their algorithms.

\section{References}

[1] M. Ludvigsen, B. Sortland, G. Johnsen, H. Singh, Applications of geo-referenced underwater photo mosaics in marine biology and archaeology, Oceanography 20 (4) (2007) 140-149. doi:10.5670/oceanog. 2007.14

[2] N. Strachan, Recognition of fish species by colour and shape, Image and Vision Computing 11 (1) (1993) 2-10. doi:10.1016/ 0262-8856 (93) 90027-E

[3] M. Costa, P. Goncalves, A. Martins, E. Silva, Vision-based assisted teleoperation for inspection tasks with a small ROV, in: Oceans, 2012, 2012, pp. 1-8. doi:10.1109/OCEANS. 2012.6404915

[4] N. Gracias, J. Santos-Victor, Underwater video mosaics as visual navigation maps, Computer Vision and Image Understanding 79 (1) (2000) 66-91. doi:10.1006/cviu.2000.0848

[5] N. Gracias, M. Mahoor, S. Negahdaripour, A. Gleason, Fast image blending using watersheds and graph cuts, Image and Vision Computing 27 (5) (2009) 597-607. doi:10.1016/j.imavis . 2008.04.014

[6] Y. Schechner, N. Karpel, Clear underwater vision, in: Proceedings of the 2004 IEEE Computer Society Conference on Computer Vision and Pattern Recognition, 2004. CVPR 2004, Vol. 1, 2004, pp. I-536-I-543 Vol.1. doi:10.1109/CVPR.2004.1315078

[7] Y. Schechner, N. Karpel, Recovery of underwater visibility and structure by polarization analysis, IEEE Journal of Oceanic Engineering 30 (3) (2005) 570-587. doi:10.1109/JOE. 2005.850871

[8] L. A. Torres-Mendez, G. Dudek, Color correction of underwater images for aquatic robot inspection, in: Proceedings of the 5th international conference on Energy Minimization Methods in Computer Vision and Pattern Recognition, EMMCVPR'05, Springer-Verlag, Berlin, Heidelberg, 2005, p. 6073. doi:10.1007/11585978_5

[9] C. Ancuti, C. Ancuti, T. Haber, P. Bekaert, Enhancing underwater images and videos by fusion, in: 2012 IEEE Conference on Computer Vision and Pattern Recognition (CVPR), 2012, pp. 81-88. doi:10.1109/CVPR.2012.6247661

[10] M. Chambah, D. Semani, A. Renouf, P. Courtellemont, A. Rizzi, Underwater color constancy: enhancement of automatic live fish recognition, in: Proc. SPIE, Vol. 5293, 2003, pp. 157-168. doi:10.1117/12.524540

[11] K. Iqbal, R. Abdul Salam, O. Mohd, T. Abdullah Zawawi, Underwater image enhancement using an integrated colour model, IAENG 32 (2) (2007) 239-244.

[12] R. Schettini, S. Corchs, Underwater image processing: State of the art of restoration and image enhancement methods, EURASIP Journal on Advances in Signal Processing 2010 (1) (2010) 746052. doi:10.1155/2010/746052 
[13] H. Koschmieder, Theorie der horizontalen Sichtweite: Kontrast und Sichtweite, Keim \& Nemnich, 1925.

[14] S. Nayar, S. Narasimhan, Vision in bad weather, in: The Proceedings of the Seventh IEEE International Conference on Computer Vision, 1999, Vol. 2, 1999, pp. 820-827 vol.2. doi:10.1109/ICCV.1999.790306

[15] D. Lvesque, F. Deschłnes, Novel depth cues from light scattering, Image and Vision Computing 27 (12) (2009) 19-36. doi:10.1016/j . imavis.2006.10.012

[16] R. Kaftory, Y. Schechner, Y. Zeevi, Variational distance-dependent image restoration, in: IEEE Conference on Computer Vision and Pattern Recognition, 2007. CVPR '07, 2007, pp. 1-8. doi:10.1109/CVPR.2007.383262

[17] K. He, J. Sun, X. Tang, Single image haze removal using dark channel prior, IEEE Transactions on Pattern Analysis and Machine Intelligence 33 (12) (2011) 2341-2353. doi:10.1109/tpami.2010.168

[18] N. Carlevaris-Bianco, A. Mohan, R. Eustice, Initial results in underwater single image dehazing, in: OCEANS 2010, 2010, pp. 1-8. doi: 10.1109/OCEANS. 2010.5664428

[19] J. Chiang, Y.-C. Chen, Underwater image enhancement by wavelength compensation and dehazing, IEEE Transactions on Image Processing 21 (4) (2012) 1756-1769. doi:10.1109/TIP.2011.2179666

[20] K. He, J. Sun, X. Tang, Guided image filtering, in: Proceedings of the 11th European Conference on Computer Vision: Part I, ECCV'10, Springer-Verlag, Berlin, Heidelberg, 2010, pp. 1-14.

[21] A. Levin, D. Lischinski, Y. Weiss, A closed-form solution to natural image matting, IEEE Transactions on Pattern Analysis and Machine Intelligence 30 (2) (2008) 228-242. doi:10.1109/TPAMI .2007.1177

[22] K. N. Plataniotis, A. N. Venetsanopoulos, Color Image Processing and Applications, Springer, 2000

[23] N. Hautiere, J.-P. Tarel, D. Aubert, E. Dumont, Blind contrast enhancement assessment by gradient ratioing at visible edges, Image Analysis \& Stereology 27 (2) (2011) 87-95. doi:10.5566/ias.v27.p87-95

[24] J. P. Tarel, N. Hautiere, Fast visibility restoration from a single color or gray level image, in: 2009 IEEE 12th International Conference on Computer Vision, 2009, pp. 2201-2208. doi:10.1109/ICCV.2009.5459251

[25] J. Malkasse, L. Jaulin, I. Quidu, S. Bazeille, Automatic underwater image pre-preprocessing, SEA TECH WEEK Caracterisation du Milieu Marin.

[26] S. Serikawa, H. Lu, Underwater image dehazing using joint trilateral filter Comput. Electr. Eng. 40 (1) (2014) 41-50. doi:10.1016/j . compeleceng.2013.10.016 URL http://dx.doi.org/10.1016/j.compeleceng.2013.10.016

[27] B. Henke, M. Vahl, Z. Zhou, Removing color cast of underwater images through non-constant color constancy hypothesis, in: 2013 8th International Symposium on Image and Signal Processing and Analysis (ISPA), 2013, pp. 20-24. doi:10.1109/ISPA.2013.6703708

[28] R. Palma-Amestoy, E. Provenzi, M. Bertalmio, V. Caselles, A perceptually inspired variational framework for color enhancement, IEEE Transactions on Pattern Analysis and Machine Intelligence 31 (3) (2009) 458-474. doi : 10.1109/TPAMI.2008.86 